\title{
Determination of Hot-Carrier Distribution Functions in Uniaxially Stressed p-Type Germanium
}

\section{Christensen, Ove}

\section{Published in:}

Physical Review B

Link to article, DOI:

10.1103/PhysRevB.7.763

Publication date:

1973

Document Version

Publisher's PDF, also known as Version of record

Link back to DTU Orbit

Citation (APA):

Christensen, O. (1973). Determination of Hot-Carrier Distribution Functions in Uniaxially Stressed p-Type Germanium. Physical Review B, 7(2), 763-777. https://doi.org/10.1103/PhysRevB.7.763

\section{General rights}

Copyright and moral rights for the publications made accessible in the public portal are retained by the authors and/or other copyright owners and it is a condition of accessing publications that users recognise and abide by the legal requirements associated with these rights.

- Users may download and print one copy of any publication from the public portal for the purpose of private study or research.

- You may not further distribute the material or use it for any profit-making activity or commercial gain

- You may freely distribute the URL identifying the publication in the public portal

If you believe that this document breaches copyright please contact us providing details, and we will remove access to the work immediately and investigate your claim 


\title{
Determination of Hot-Carrier Distribution Functions in Uniaxially Stressed $p$-Type Germanium
}

\author{
Ove Christensen* \\ Physics Laboratory III, Technical University of Denmark, Lyngby, Denmark \\ (Received 29 February 1972)
}

\begin{abstract}
This paper gives a description of an experimental determination of distribution functions in $\overrightarrow{\mathrm{k}}$ space of hot holes in uniaxially compressed germanium. The hot-carrier studies were made at $85^{\circ} \mathrm{K}$ at fields up to $1000 \mathrm{~V} / \mathrm{cm}$ and uniaxial stresses up to $11800 \mathrm{~kg} / \mathrm{cm}^{2}$. The field and stress were always in the $\langle 111\rangle$ direction. For the highest stresses, the maximum fields were close to the threshold for current oscillations. The distribution functions were obtained from experimental modulation of intervalence-band absorption of infrared radiation. In order to interpret the results, a parametrized distribution function has been assumed. The parameters of the distribution function are then fitted to the experimental modulation. The calculation of absorption was performed numerically, using a four-band $\vec{k} \cdot \vec{p}$ model. This model was checked for consistency by comparing with piezoabsorption measurements performed in thermal equilibrium. The average carrier energy calculated from the distribution function shows a fast increase with stress and almost saturates when the strain splitting of the two $p_{3 / 2}$ levels reaches the optical-phonon energy. This saturation is interpreted in terms of the change in scattering probabilities with stress. A model based on the nonparabolicity of the upper $p_{3 / 2}$ level is proposed for the negative differential conductivity in stressed $p$-type Ge.
\end{abstract}

\section{INTRODUCTION}

The hot-carrier properties of $p$-type germanium have previously been studied by means of modulation of the infrared absorption due to intervalenceband transitions of free holes. ${ }^{1-6}$ Baynham and Paige ${ }^{4,6}$ found that the distribution function of the hot carriers was characterized by different temperatures for carriers with energies smaller or larger than the optical-phonon energy. Pinson and Bray $^{5}$ determined energy distribution functions at different fields and analyzed the energy-loss rates of carriers to optical and acoustic phonons. The measurements of Refs. 1-6 have demonstrated the dominant role of the interaction between hot holes and optical phonons in Ge.

A particularly interesting aspect of hot-carrier properties in $p$-type Ge is the case of a high uniaxial stress in the direction of the electric field. Under these conditions, $p$-type Ge exhibits bulk negative differential conductivity (BNDC) at sufficiently high fields. ${ }^{8-10}$ Because of the extremely complicated valence-band structure, several mechanisms can be responsible for the BNDC. Ridley and Watkins ${ }^{11}$ initially considered the process of carrier transfer between the two upper strain-split valleys of the valence band. ${ }^{12}$ The nonparabolicity of the upper band might however also be responsible for the occurrence of BNDC. ${ }^{13}$

The purpose of the present work is to extend studie $^{1-6}$ of the distribution function of hot holes in Ge to the case of a high uniaxial stress applied parallel to the electric field. For experimental convenience the present investigation is limited to liquid-nitrogen temperature. Here a BNDC occurs only for a stress in the $\langle 111\rangle$ direction ${ }^{9,10}$ and we therefore choose this condition for the experiments. As in previous works on unstressed material, the experimental method of the study is electroabsorption, i.e., the modulation of infrared absorption by a pulsed uniform electric field. In this way, we are restricted to measurements at electric fields below the threshold field for current oscillations. This field is strongly dependent on the magnitude of the uniaxial stress. ${ }^{9,10}$ In order to get information about the mechanism of the Gunn effect, ${ }^{14}$ we present measurements at different electric fields and different uniaxial stresses. This allows us to separate stress-dependent and field-dependent properties of the hot carriers.

In unstressed material, Pinson and $\mathrm{Bray}^{5}$ assumed spherical bands which establishes a one-toone relationship between carrier energies in the heavy - and light-hole bands and corresponding photon energies. The valence-band structure of uniaxially stressed Ge is very complex. It is no longer possible to associate a given carrier energy with a definite photon energy. It is, however, possible to calculate the intervalence-band absorption due to a given distribution function of holes. holes. ${ }^{15,16}$ We shall therefore approach the problem of finding the hole distribution function from measurements of electroabsorption in a computational way. The distribution function of the hot holes is approximated by an analytical expression, containing several parameters. The electroab- 
sorption spectrum is calculated from this distribution function and the parameters are adjusted to give agreement with experiment.

Calculation of the absorption from intervalenceband transitions requires a rather sophisticated $\vec{k} \cdot \vec{p}$ treatment of the valence-band levels to give agreement with experiments. ${ }^{16,19-21}$ As a check on our computational model at high uniaxial stress, we have performed measurements of piezoabsorption at high uniaxial stresses in thermal equilibrium.

In Sec. II, the computation of absorption from intervalence-band transitions is briefly summarized. The computational model is checked by comparison with piezoabsorption measurements presented in Sec. III. Section IV gives the details of the experimental procedure and Sec. V contains the experimental electroabsorption results together with a qualitative interpretation.

In Sec. VI, we give a description of the analytical distribution function. Section VII contains some applications of the distribution functions together with an interpretation of some hot-carrier properties. On this basis, the BNDC occurring in uniaxially stressed $p$-type Ge is discussed. Finally, we propose a simple model for the BNDC based essentially on the nonparabolicity of the band structure.

\section{CALCULATION OF INTERVALENCE-BAND ABSORPTION}

In this section we give an outline of the calculation of absorption due to momentum-conserving transitions of free holes between pairs of the three valence-band sublevels of germanium. The levels are denoted by index $i$, where $i=1,2$, and 3 correspond to the heavy-hole band, the light-hole band, and the spin-orbit split-off band, respectively.

When a uniaxial stress is applied, index 1 and 2 denote the upper and lower of the strain-split $p_{3 / 2}$ levels. For electromagnetic radiation of frequency $\omega$ and unit polarization vector $\overrightarrow{\dot{e}}$, the contribution to the absorption due to transitions from band $i$ to band $j$ is given by ${ }^{22}$

$$
\begin{aligned}
\alpha_{i j}=(p c / n \hbar \omega) \int d \overrightarrow{\mathrm{k}} & \left|M_{i j}(\overrightarrow{\mathrm{k}})\right|^{2}\left(f_{i}-f_{j}\right) \\
& \times \delta\left(\epsilon_{i}(\overrightarrow{\mathrm{k}})-\epsilon_{j}(\overrightarrow{\mathrm{k}})-\hbar \omega\right),
\end{aligned}
$$

where $p$ is the concentration of holes, $c$ a constant, $n$ the refractive index, and

$$
M_{i j}(\overrightarrow{\mathrm{k}})=\langle j|\overrightarrow{\mathrm{e}} \cdot \overrightarrow{\mathrm{p}}| i\rangle
$$

is the optical matrix element for $i-j$ transitions at hole wave vector $\overrightarrow{\mathrm{k}}$. $f_{i}$ and $f_{j}$ are normalized occupation probabilities for holes with energies $\epsilon_{i}(\overrightarrow{\mathrm{k}})$ and $\epsilon_{j}(\overrightarrow{\mathrm{k}})$. In thermal equilibrium at temperature $T$ for nondegenerate materials, $f_{i} \propto e^{\epsilon_{i}(\vec{k}) / k_{B} T}$ $\left[\epsilon_{i}(\overrightarrow{\mathrm{k}})<0\right]$, where $k_{B}$ is the Boltzmann constant.
Since the spin-orbit splitting of the valence-band levels is $0.295 \mathrm{eV}$ in $\mathrm{Ge}$, we have in the temperature range of interest $f_{3} \simeq 0$. In this work we shall only be interested in 1-3 and 2-3 transitions. The valence-band calculations of Fawcett ${ }^{7}$ have shown that the absorption bands arising from these two types of transitions do not overlap at temperatures of interest here. This turns out to be still true also when an obtainable uniaxial stress is applied. The absorption at photon energies of interest here, $0.26-0.70 \mathrm{eV}$, is then entirely due to $1-3$ and $2-3$ transitions.

The procedure for numerical computation of the absorption constant was, except for minor details, performed as described by Arthur, Baynham, Fawcett, and Paige (ABFP). ${ }^{21}$ In our $\vec{k} \cdot \vec{p}$ treatment of the valence-band energies, we have taken into account the twofold degenerate Kramers doublets of the three $\Gamma_{25}^{\prime}$ valence-band levels together with the $\overrightarrow{\mathrm{k}}=0$ conduction-band state $\Gamma_{2}^{\prime}$. The energies of these states were found by diagonalizing $8 \times 8$ Hermitian matrices. The $\overrightarrow{\mathrm{k}} \cdot \overrightarrow{\mathrm{p}}$ matrix elements were the $k$-linear terms ${ }^{7}$ between the $\Gamma_{25}^{\prime}$ and $\Gamma_{2}^{\prime}$ states and $k^{2}$ elements, ${ }^{20,23}$ giving the interaction between the valence-band levels through intermediate states except the $\Gamma_{2}^{\prime}$ state. ${ }^{16}$ Our $\overrightarrow{\mathrm{k}} \cdot \overrightarrow{\mathrm{p}}$ model is similar to Balslev's four-band model ${ }^{16}$ but no approximations were made in the present calculation. The effect of strain was included in the matrix elements between the valence bands. ${ }^{12}$ The value of the energy separation $\Gamma_{25}^{\prime}-\Gamma_{2}^{\prime}$ was adjusted according to the known variation with the dilatational part of the uniaxial stress. Table I displays the parameters of the calculation. The optical matrix elements between the $\overrightarrow{\mathrm{k}}=0$ basis functions were found as described by Kane. ${ }^{20}$ At given $\vec{k}$, the optical matrix elements were found by proper transformation to the diagonal representation at this point.

The calculation of the absorption constant for 1-3 and 2-3 transitions was done by summing the contributions to the integral (1) over a mesh of points in $\overrightarrow{\mathrm{k}}$ space. The number of points used was 12000-25000. At the first run, the calculated hole energies, photon energies and optical matrix elements at each point of the mesh were written out on magnetic tape. The absorption constant could be then generated from this tape in around $0.5 \%$ of the time needed for the initial calculation. The absorption constant was obtained in the form of a histogram to which a Gaussian broadening ${ }^{6}$ was applied. The standard deviation of this was $8.6 \mathrm{meV}$ at $85^{\circ} \mathrm{K}$ and $15 \mathrm{meV}$ at $300^{\circ} \mathrm{K}$ except for the calculation of electroabsorption spectra where a broadening of $20 \mathrm{meV}$ was applied to account for the experimental resolution.

It is useful to see how close a relationship there exists between the absorption at given photon ener- 
TABLE I. Parameters used in calculation of the absorption of $p$-type Ge.

\begin{tabular}{|c|c|c|c|c|}
\hline Quantity & Symbol & Value at $77^{\circ} \mathrm{K}$ & Value at $300^{\circ} \mathrm{K}$ & Unit \\
\hline Valence-band parameter & $\begin{array}{l}A \\
B \\
N\end{array}$ & $\begin{array}{r}-13.35^{\mathrm{a}} \\
-8.50^{\mathrm{a}} \\
-34.14^{\mathrm{a}}\end{array}$ & $\begin{array}{r}-13.35 \\
-8.50 \\
-34.14\end{array}$ & $\hbar^{2} / 2 m_{0}$ \\
\hline Energy separation $\Gamma_{25}^{\prime}-\Gamma_{2}^{\prime}$ & $E_{0}$ & $0.89^{\mathrm{b}}$ & $0.80^{\mathrm{b}}$ & $\mathrm{eV}$ \\
\hline Momentum matrix element & $p^{2}$ & $26.3^{\mathrm{c}}$ & 26.3 & $\mathrm{eV} \times\left(\hbar^{2} / 2 m_{0}\right)$ \\
\hline Valence-band deformation potential ${ }^{d}$ & $b$ & $\begin{array}{l}-2.6^{\ominus} \\
-7.6^{\ominus}\end{array}$ & $\begin{array}{l}-2.3^{\ominus} \\
-7.1^{\ominus}\end{array}$ & \multirow[t]{2}{*}{$\mathrm{eV}$} \\
\hline Dilatational deformation potential for $E_{0}$ & $d_{0}$ & $-10.6^{f}$ & -10.6 & \\
\hline 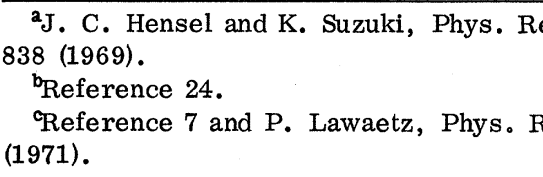 & $\begin{array}{l}\text { ters } \underline{22}, \\
3460\end{array}$ & $\begin{array}{l}{ }^{\mathrm{d}} \text { In the nota } \\
{ }^{\text {e}} \text { Reference } \\
{ }^{\mathrm{P}} \mathrm{P} . \mathrm{J} . \mathrm{Mel} \\
(1971) \text {. }\end{array}$ & $\begin{array}{l}\text { Ref. } 12, d=n / \sqrt{ } \\
\text { Ortenburger, }\end{array}$ & ev. B 3,3257 \\
\hline
\end{tabular}
(1971).

gy and the concentration of carriers at given hole energy. One way to examine this is to calculate the absorption from a monoenergetic distribution function. In Figs. 1 and 2 we show such spectra for zero stress (Fig. 1) and for a uniaxial stress of $11800 \mathrm{~kg} / \mathrm{cm}^{2}$ in the $\langle 111\rangle$ direction (Fig. 2)。 In these figures $\sigma_{\|}$and $\sigma_{\perp}$ denote the absorption cross section for light polarized parallel and perpendicular to field (and stress). The direction of polarization is not important in this connection. For no stress, there exists some correspondence between initial hole energy and photon energy but at high uniaxial stress, it is no longer possible to establish a connection between photon and hole energy. The only thing still being true is that the absorption at large photon energies is due to holes with high energy. The distribution function can, therefore, not be determined uniquely. Rather, the parameters of an assumed distribution function can be fit.

The absorption constant of Eq. (1) is at any wavelength a functional of the hole distribution function. The latter is $f_{T}(\overrightarrow{\mathrm{k}})$ in thermal equilibrium at lattice temperature $T$ and $f_{E}(\overrightarrow{\mathrm{k}})$ when an electric field is applied. The corresponding absorption is $\alpha^{T}(\hbar \omega)$ $=\alpha\left(\hbar \omega, f_{T}\right)$ and $\alpha^{E}(\hbar \omega)=\alpha\left(\hbar \omega, f_{E}\right)$. The modulation of absorption due to an electric field is then given by

$$
\Delta \alpha=\alpha^{E}-\alpha^{T}
$$

which is usually different for light polarized parallel and perpendicular to the electric-field direction. The interpretation of electroabsorption spectra can be done by assuming an analytical form of the distribution function $f_{E}(\overrightarrow{\mathrm{k}})$ containing some parameters. $\Delta \alpha$ is calculated for the two polarizations of light parallel and perpendicular to the field, and the parameters are adjusted until satisfactory agreement with experimental data is obtained.

\section{RESULTS FROM PIEZOABSORPTION MEASUREMENTS}

The computational procedure has been tested at zero stress by comparing our calculations of the differential absorption due to a change in lattice temperature from 77 to $92.5^{\circ} \mathrm{K}$ with the exact

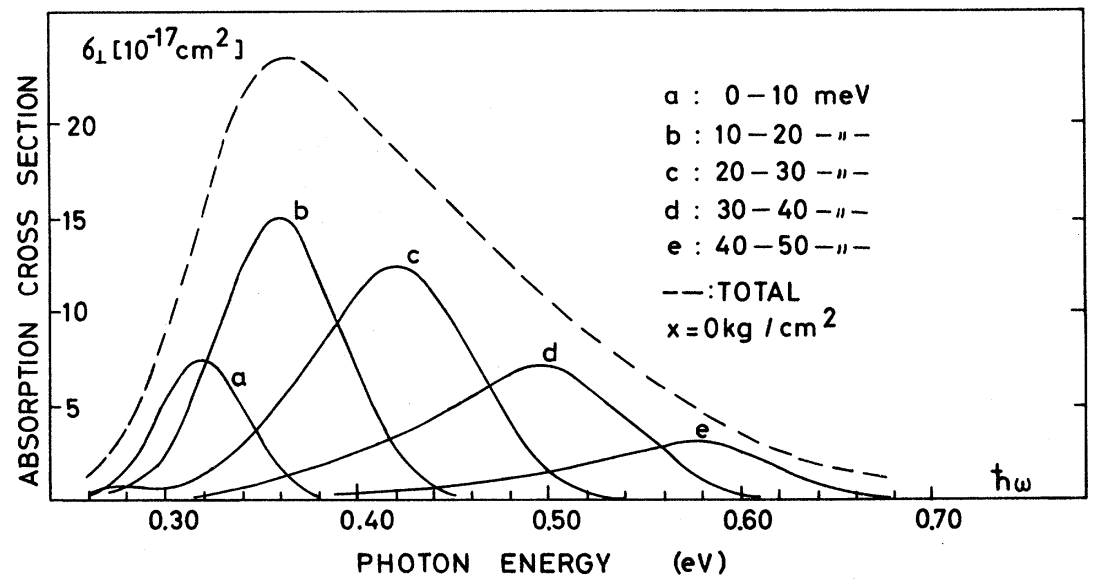

FIG. 1. Absorption spectra from "monoenergetic" distribution functions at zero stress. The distribution function is the one found at 500 $\mathrm{V} / \mathrm{cm}$ for hole energies in the indicated intervals, zero outside. 


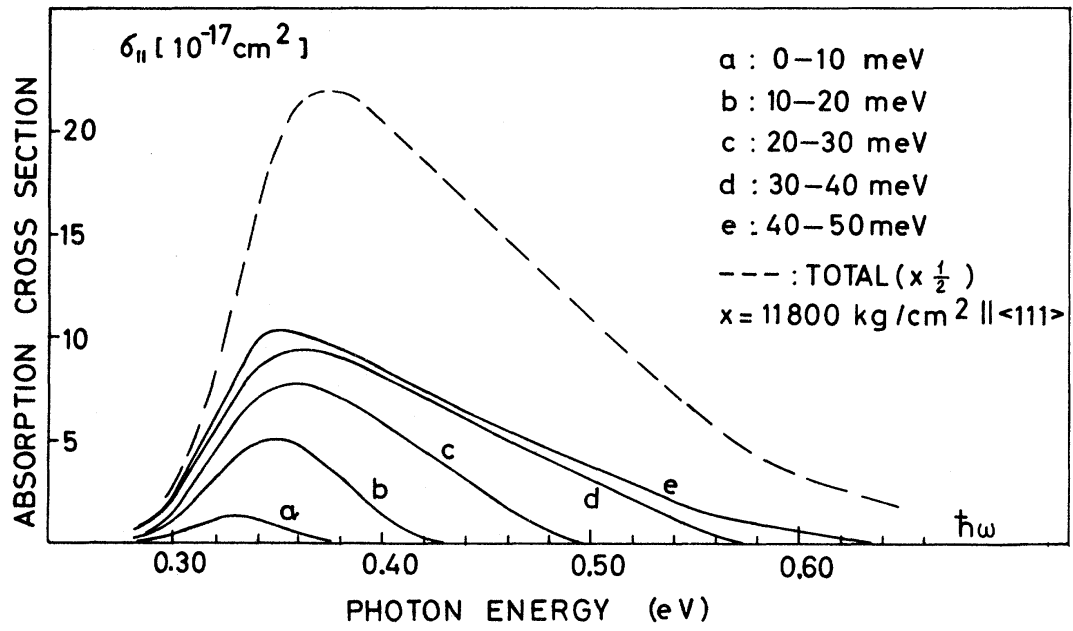

FIG. 2. As Fig. 1 but for a stress of $11800 \mathrm{~kg} / \mathrm{cm}^{2}$ in the $\langle 111\rangle$ direction, $E=500 \mathrm{~V} / \mathrm{cm}$.

seven-band calculation of ABFP. With identical band parameters, the agreement was excellent. In order to make a check when a high uniaxial stress is applied, we have performed measurements and calculations of the absorption in thermal equilib-

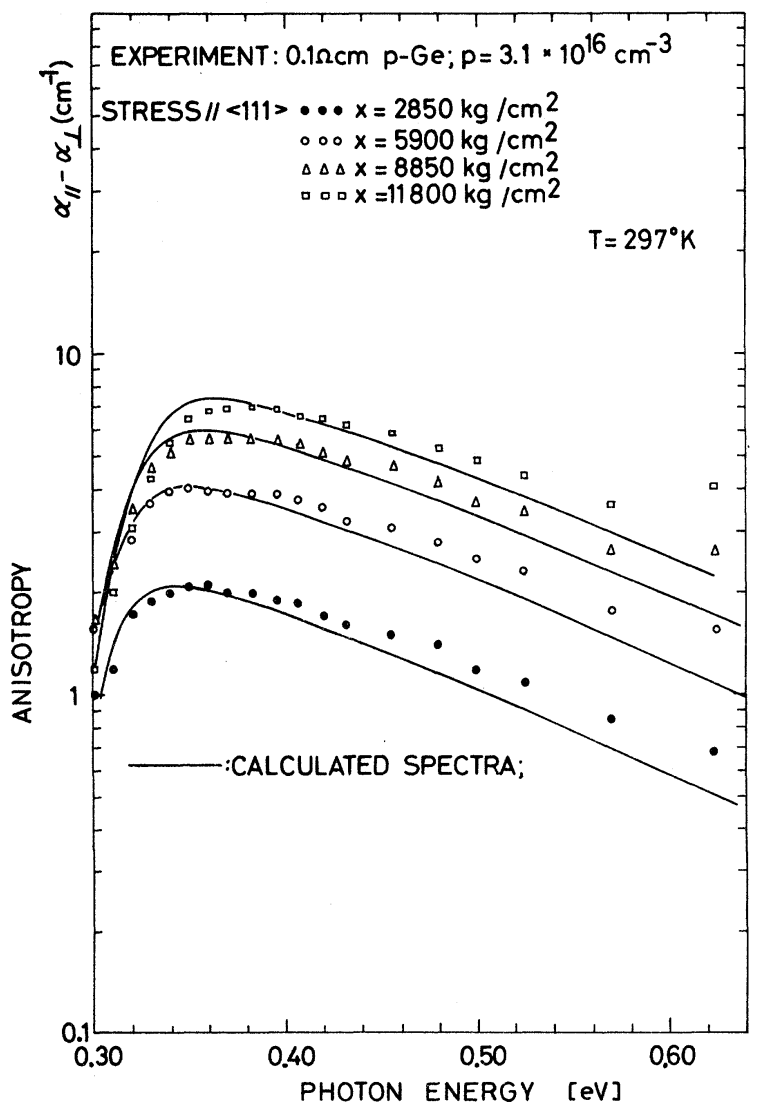

FIG. 3. Experimental and calculated dichroism of $p$ type Ge at $297^{\circ} \mathrm{K}$ for uniaxial stresses in the $\langle 111\rangle$ direction. rium at 85 and $300{ }^{\circ} \mathrm{K}$ for different magnitudes of uniaxial stress.

When a uniaxial stress is applied, complicated changes in the energies of the $p_{3 / 2}$ levels occur. Repopulation of the hole states and changes in optical matrix elements give rise to an absorption which depends on the direction of polarization of the incoming light with respect to the stress axis. For a compressive stress it turns out that in the region of 1-3 transitions the absorption constant for light polarized parallel to stress, $\alpha_{\| 1}$, becomes larger than the absorption constant for light polarized perpendicular to stress, $\alpha_{\perp}{ }^{15}$ The dichroism $\alpha_{11}-\alpha_{\perp}$ is usually the experimentally determined quantity. ${ }^{15,16}$ In Fig. 3 we show the experimental and calculated dichroism at room temperature for several values of uniaxial stress in the $\langle 111\rangle$ direction. Figure 4 displays the same quantities at $85^{\circ} \mathrm{K}$. A discussion of similar results at low stresses has been given in Refs. 15 and 16. The main difference between high- and low-stress results is that at $85^{\circ} \mathrm{K}$ a saturation of the dichroism occurs and that increasing stress shifts the spectrum towards higher photon energies. This shift is due to the increase of the $1-3$ separation at $\vec{k}=0$ with stress. At $85^{\circ} \mathrm{K}$ for some strain splitting of the $p_{3 / 2}$ levels, most carriers occupy the highly perturbed region around $\overrightarrow{\mathrm{k}}=0$ with energies smaller than the strain splitting. An increase of stress changes the band structure only outside this region (apart from a common energy shift). Within the occupied region of $\vec{k}$ space the optical matrix elements are independent of stress. Except for an energy displacement, the absorption becomes independent of stress at high stress.

Generally, the agreement between experiment and theory is rather satisfying. Also, the lowstress measurements are in accordance with Balslev's ${ }^{16}$ results. The small disagreements at 


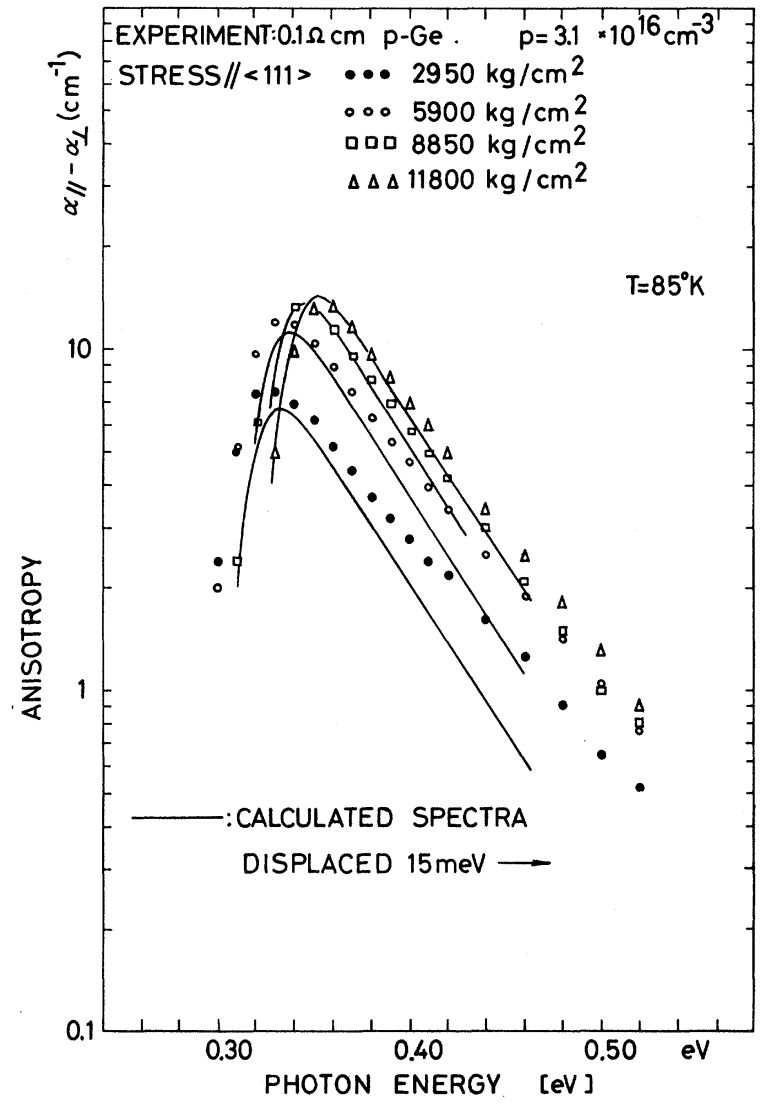

FIG. 4. Experimental and calculated dichroism of $p$ type Ge at $85^{\circ} \mathrm{K}$ for uniaxial stresses in the $\langle 111\rangle$ direction.

high photon energies are almost the same at the two temperatures and they, therefore, are not expected to contribute to the modulated spectra. Similar measurements for $\langle 100\rangle$ stress gave very good agreement with calculated spectra at room temperature, but somewhat worse agreement at $85^{\circ} \mathrm{K}$. To within $10 \%$, these experiments have confirmed the values of the deformation potentials $b$ and $n$ listed in Table I. The only serious disagreement between theory and experiment is that the calculated maximum of dichroism occurs at 10-15 meV lower photon energy than the experimental value. The reason for this is not understood. A similar disagreement was found in Refs. 15 and 16 . It is puzzling that it is not necessary to introduce any corresponding energy displacement in the calculations of the electroabsorption spectra to obtain good agreement with experiment.

\section{EXPERIMENTAL PROCEDURE}

In order to prepare the samples, oriented wafers of $p$-type Ge were cut from single-crystal ingots using a diamond saw. The wafers were lapped and polished to a thickness of $2.7 \mathrm{~mm}$, flat within 1 $\mu \mathrm{m}$ in $1 \mathrm{~cm}$ and plane parallel within $5 \times 10^{-4} \mathrm{rad}$. The samples were next cut, ground, and polished to a dimension of $1.0 \times 1.0 \times 2.7 \mathrm{~mm}^{3}$, the $2.7 \mathrm{~mm}$ being the stress and/or field direction. For the samples to be used in measurements of modulated absorption, ohmic contacts were formed by electroplating the wafers with $\mathrm{Au}^{10}$

The experimental setup is shown in Fig. 5. The sample is squeezed between two plane and parallel tungsten-carbide discs, which at the same time form the electrical contacts. One of these discs is insulated from the Dewar by a sapphire disc. ${ }^{9,10}$ The stress is applied to the pressure piston through a pulling frame. The fracture stress for such samples was $1.2-1.5 \times 10^{4} \mathrm{~kg} / \mathrm{cm}^{2}$ in this apparatus. The whole pressure system was situated in vacuum. The temperature of the sample was $85^{\circ} \mathrm{K}$ with liquid nitrogen in the Dewar.

Light from a globar was passed through a wiregrid polarizer, focused on the sample, and analyzed by a Perkin Elmer 98 monochromator, equipped with a $\mathrm{NaCl}$ prism. The spectral resolution at $\hbar \omega=0.40 \mathrm{eV}$ was $10 \mathrm{meV}$ in the measurements of piezoabsorption and $20 \mathrm{meV}$ in the measurements of modulated absorption. The detector was an InSb photovoltaic cell (Philco ISC-301D) with a rise time less than $1 \mu \mathrm{sec}$.

In the measurements of modulated absorption, the electrical pulses to the sample were supplied by a $25-\Omega$-discharge-line pulse generator. The pulsed change in detector signal was amplified and detected by a PAR Model No. 160 boxcar integrator, using a gate width of $0.5 \mu \mathrm{sec}$. Under these circumstances, it was found that a duration of the electrical pulse of $2 \mu \mathrm{sec}$ was necessary for the signal not to be affected by the detector time con-

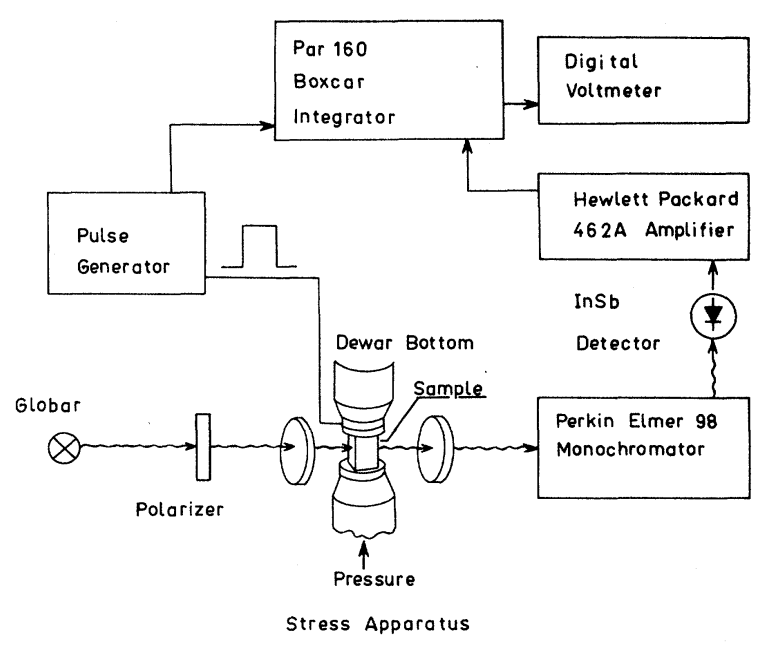

FIG. 5. Experimental setup for measurements of field-modulated absorption in uniaxially stressed $p$-type Ge. 


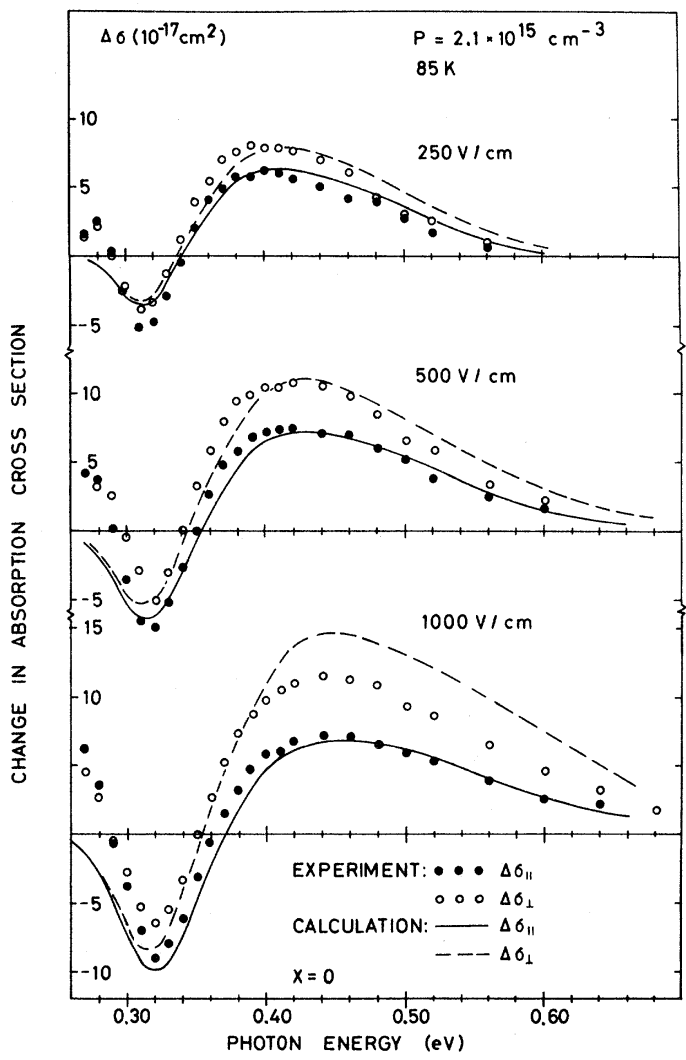

FIG. 6. Field-induced change in absorption cross section of $p$-type Ge at zero stress. Electric field in the $\langle 111\rangle$ direction.

stant. The effect of heating of the sample during the electrical pulse will be considered in Sec. V. With a repetition frequency of $50 \mathrm{~Hz}$ and a time constant of $15 \mathrm{sec}$ for the boxcar integrator, a relative change in transmission of $4 \times 10^{-4}$ could be measured. The change in absorption $\Delta \alpha$ is found from the change in intensity during the pulse $\Delta I$ and the measured intensity of transmitted light without field $I_{T}$. For a carrier concentration of $p$ $=2.1 \times 10^{15} \mathrm{~cm}^{-3}$ and a sample thickness of $0.1 \mathrm{~cm}$, both $\alpha d$ and $\Delta \alpha d$ were less than 0.1 , and $\Delta \alpha$ can be found directly from the relation $\Delta \alpha d \simeq \Delta I / I_{T}$. The measured $\Delta \alpha$ is normalized to unit carrier concentration to give the change in absorption cross section $\Delta \sigma=\Delta \alpha / p$.

In the measurement of piezoabsorption, a chopper was introduced after the globar and the signal from the detector was phase sensitively detected. The reduction of data from piezoabsorption measurements was done by measuring the intensity ratio for light polarized parallel and perpendicular to the stress direction. This was first done at zero stress $I_{11}^{0} / I_{\perp}^{0}$ and next for an applied uniaxial stress $I_{\|}^{s} / I_{\perp \bullet}^{s}$ Let $d$ be the sample thickness, $R$ the reflection, and $\alpha_{11}$ and $\alpha_{\perp}$ the absorption for the two polarizations. Then

$$
\frac{I_{\|}^{s}}{I_{\perp}^{s}} \frac{I_{1}^{0}}{I_{\|}^{0}}=e^{-\delta \alpha d}=e^{-\left(\alpha_{\|}-\alpha_{\perp}\right) d}\left(\frac{1-R^{2} e^{-2 \alpha_{\perp} d}}{1-R^{2} e^{-2 \alpha_{\|} \|^{d}}}\right) \text {. }
$$

The last factor gives only a contribution of $\simeq 5 \%$ to $\delta \alpha d$ and it was therefore calculated from the computed values of $\alpha_{11}$ and $\alpha_{1}$.

\section{RESULTS FROM ELECTROABSORPTION EXPERIMENT}

Electroabsorption experiments have been performed on $p$-type Ge at $85^{\circ} \mathrm{K}$ for different values of uniaxial stress and electric field. The stresses were $0,5900,8850$, and $11800 \mathrm{~kg} / \mathrm{cm}^{2}$ along the $\langle 111\rangle$ direction. The corresponding splittings between the $p_{3 / 2}$ bands at $\vec{k}=0$ are $0,22.3,35.6$, and $45.9 \mathrm{meV}$. At the three first stresses, measurements were performed at 250, 500, and 1000 $\mathrm{V} / \mathrm{cm}$. At $11800 \mathrm{~kg} / \mathrm{cm}^{2}$, Gunn effect was observed at $570 \mathrm{~V} / \mathrm{cm}$. The fields used here were 100, 250, and $500 \mathrm{~V} / \mathrm{cm}$. The electric field was always in the stress direction. All measurements were done on material with a hole concentration of $2.1 \times 10^{15} \mathrm{~cm}^{-3}$ and the results obtained were reproducible within $10 \%$ from sample to sample. The results are

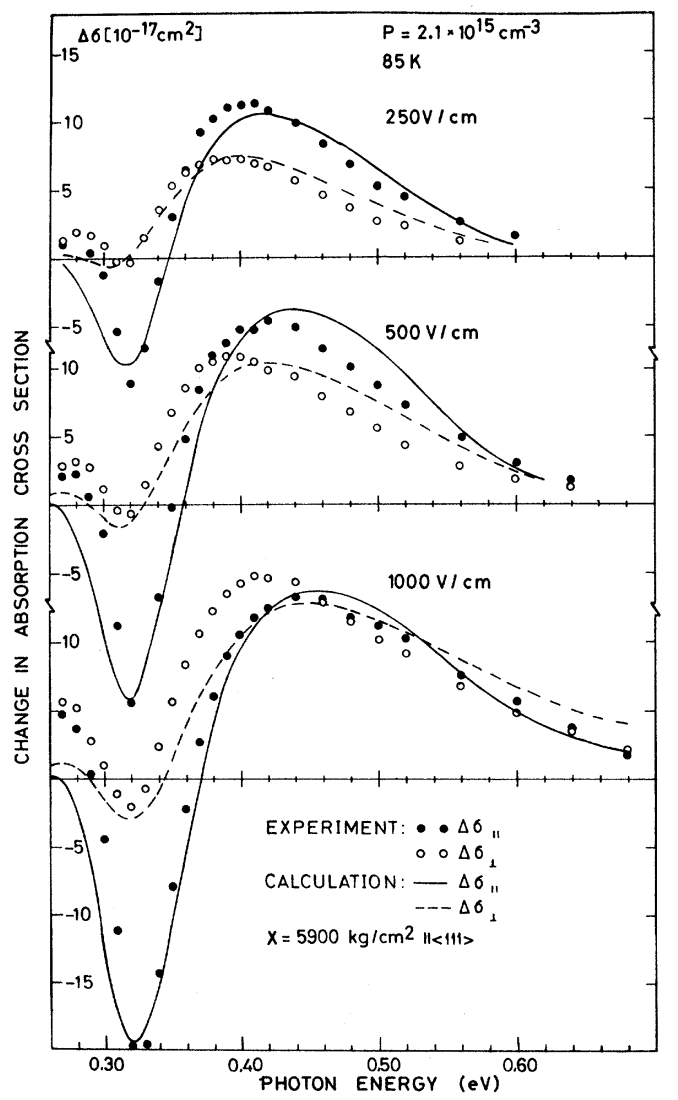

FIG. 7. Field-induced change in absorption cross section of $p$-type Ge at a uniaxial stress of $5900 \mathrm{~kg} / \mathrm{cm}^{2}$. Field and stress in the $\langle 111\rangle$ direction. 


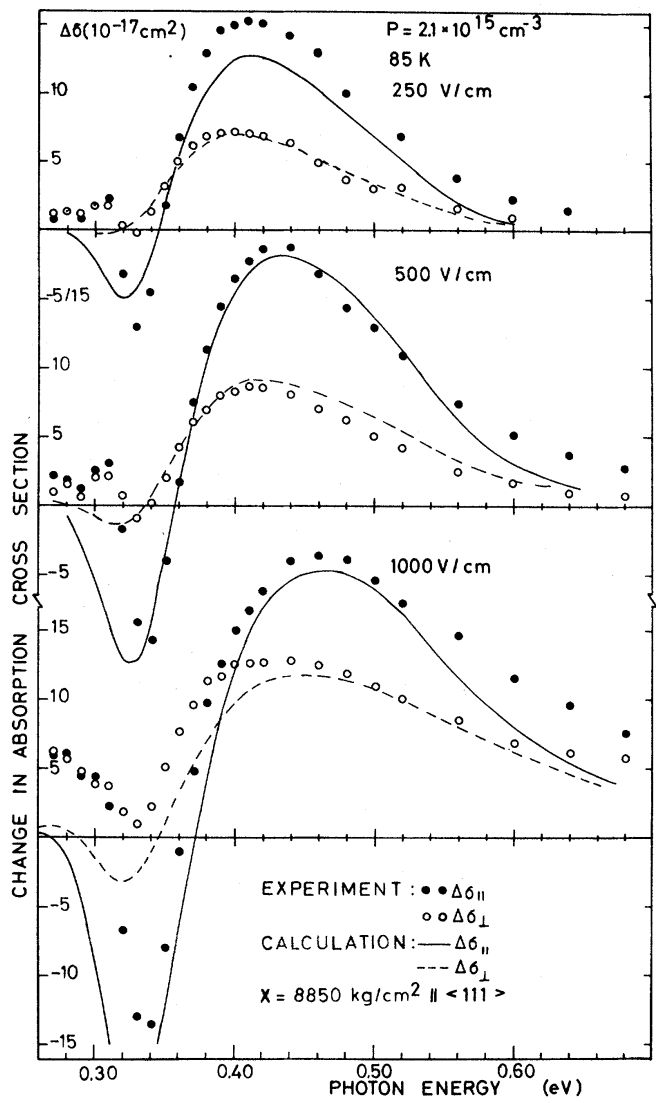

FIG. 8. Same as Fig. 7 at a stress of $8850 \mathrm{~kg} / \mathrm{cm}^{2}$.

shown in Figs. 6-9 in terms of the change in absorption cross section for light polarized parallel and perpendicular to the $\langle 111\rangle$ direction $\Delta \sigma_{\|}$and $\Delta \sigma_{\perp}$, respectively. Using $\alpha^{E}$ and $\alpha^{T}$ defined in Sec. II, the measured quantities are

$$
\begin{aligned}
& \Delta \sigma_{\|}=\left(\alpha_{\|}^{E}-\alpha_{\| 1}^{85^{\circ} \mathrm{K}}\right) / p, \\
& \Delta \sigma_{\perp}=\left(\alpha_{\perp}^{E}-\alpha_{\perp}^{85^{\circ} \mathrm{K}}\right) / p .
\end{aligned}
$$

In general, $\Delta \sigma_{\|}$and $\Delta \sigma_{\perp}$ are different. This is referred to as the anisotropy of the spectra since it reflects the anisotropy of the distribution function in $\vec{k}$ space. The general features of the spectra for zero stress have been discussed in the literature. ${ }^{3,4}$ In this section we shall only give a qualitative explanation of the relationship between distribution function and the observed modulation of the absorption.

We consider first the region of 1-3 transitions which extends from approximately $0.29 \mathrm{eV}$ to the cutoff from the band edge. The distribution function in $\vec{k}$ space can be correlated with the absorption spectrum through the transition probability for 1-3 transitions. In Fig. 10 is shown the angular variation of this quantity for light polarized paral- lel and perpendicular to the $\langle 111\rangle$ direction $w_{11}(\overrightarrow{\mathrm{k}})$ and $w_{\perp}(\overrightarrow{\mathrm{k}})$, respectively. The electric field tends to concentrate the carriers in the direction of field where at zero stress $w_{\perp}(\overrightarrow{\mathrm{k}})>w_{\|}(\overrightarrow{\mathrm{k}})$. Accordingly, $\Delta \sigma_{\perp}>\Delta \sigma_{\|}$over most of the spectral region; the anisotropy of the spectra increasing with field. At high fields, the number of carriers with small energies decrease and more carriers obtain high energies. From Fig. 1, high hole energies correspond to high photon energies. The absorption, therefore, increases at high photon energies and decreases at low photon energies as seen in Fig. 6 .

When a uniaxial stress is applied, the band structure close to $\overrightarrow{\mathrm{k}}=0$ becomes highly perturbed as shown in Fig. 11. For large wave vectors the energies are lowered parallel to stress and increased perpendicular to stress, compared with unstressed material. In thermal equilibrium most of the carriers occupy a region in $\overrightarrow{\mathrm{k}}$ space perpendicular to the stress direction. Figure 10(b) shows that in the region close to $\overrightarrow{\mathrm{k}}=0$, we always have $w_{\mathrm{II}}(\overrightarrow{\mathrm{k}})>w_{\perp}(\overrightarrow{\mathrm{k}})$ and that the transition probability is unaltered with stress at large wave vectors. Most of the carriers then experience a situation where $w_{11}(\overrightarrow{\mathrm{k}})>w_{\perp}(\overrightarrow{\mathrm{k}})$. This produces $\alpha_{\| 1}(\hbar \omega)>\alpha_{\perp}(\hbar \omega)$ for the

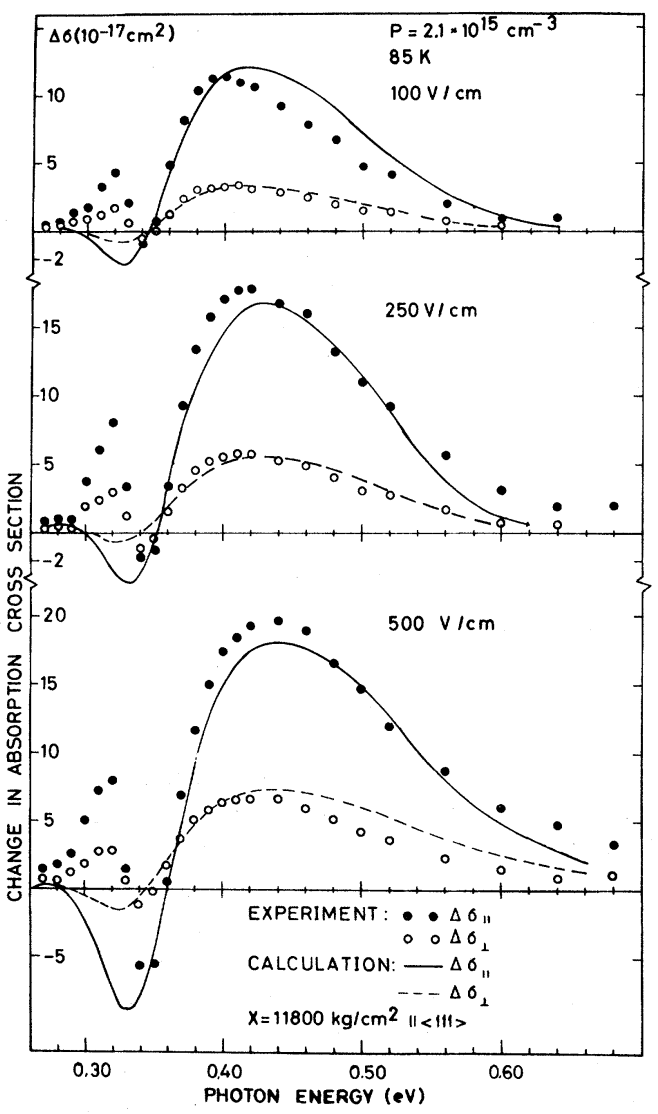

FIG. 9. Same as Fig. 7 at a stress of $11800 \mathrm{~kg} / \mathrm{cm}^{2}$. 

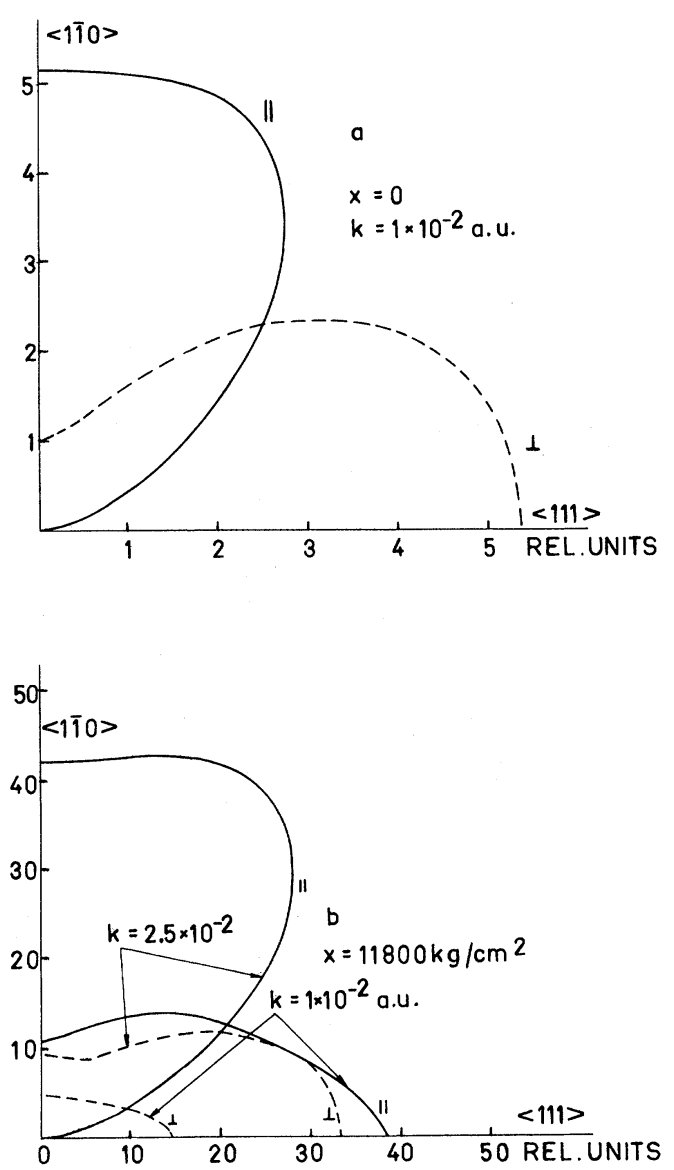

FIG. 10. Angular variation of the transition probabilities $w_{\| 1}(\overrightarrow{\mathrm{k}})$ and $w_{\perp}(\overrightarrow{\mathrm{k}})$. Plotted for fixed wave vector vs angle in a plane containing the $\langle 111\rangle$ and $\langle 1 \overline{1} 0\rangle$ axes: (a) zero stress; (b) stress of $11800 \mathrm{~kg} / \mathrm{cm}^{2}$ along the $\langle 111\rangle$ direction.

region of 1-3 transitions. For small fields we get, therefore, $\Delta \sigma_{\|}>\Delta \sigma_{\perp}$. When at high fields the carriers tend to occupy the region in $\overrightarrow{\mathrm{k}}$ space parallel to field and stress, there will be a competition between the trend to have $\Delta \sigma_{\|}>\Delta \sigma_{\perp}$ due to properties of the band structure, and to have $\Delta \sigma_{1}>\Delta \sigma_{\|}$due to the influence of the electric field. This is most clearly demonstrated in Fig. 7 for a stress of $5900 \mathrm{~kg} / \mathrm{cm}^{2}$. At $250 \mathrm{~V} / \mathrm{cm}, \Delta \sigma_{\|}>\Delta \sigma_{\perp}$ and at 1000 $\mathrm{V} / \mathrm{cm}, \Delta \sigma_{\|}<\Delta \sigma_{\perp}$ at the maximum of the differential absorption. The absorption in the region of photon energies below $\simeq 0.29 \mathrm{eV}$ is the contribution from band 2 , the lower of the strain-split $p_{3 / 2}$ bands. At $5900 \mathrm{~kg} / \mathrm{cm}^{2}$, this band gives a significant contribution to the absorption at all fields used. At $8850 \mathrm{~kg} / \mathrm{cm}^{2}$ the effect is only present at $1000 \mathrm{~V} /$ $\mathrm{cm}$ and it is not present at $11800 \mathrm{~kg} / \mathrm{cm}^{2}$.

An increase of lattice temperature during the electrical pulse will also produce a change in absorption ${ }^{21}$ similar to the measured electroabsorption. For a pulse duration of $2 \mu \mathrm{sec}$ and a carrier concentration of $2.1 \times 10^{15} \mathrm{~cm}^{-3}$, the mean temperature shift will be $6{ }^{\circ} \mathrm{K}$ at the maximum power input, i.e., at $8850 \mathrm{~kg} / \mathrm{cm}^{2}$ for $1000 \mathrm{~V} / \mathrm{cm}$. The corresponding change in absorption cross section ${ }^{21}$ is at zero stress $\Delta \sigma \simeq 1 \times 10^{-17} \mathrm{~cm}^{2}$ at $\hbar \omega=0.40 \mathrm{eV}$ and $\Delta \sigma \simeq 0.2 \times 10^{-17} \mathrm{~cm}^{2}$ at $\hbar \omega=0.60 \mathrm{eV}$. This effect is therefore negligible compared to the field-induced changes in absorption cross section. More serious is the thermal modulation of the band edge. A $16{ }^{\circ} \mathrm{K}$ increment of lattice temperature ${ }^{24}$ produces a change in absorption of $\simeq 0.1 \mathrm{~cm}^{-1}$ for $\hbar \omega$ $=0.70$ to $0.76 \mathrm{eV}$. With the actual carrier concentration the corresponding change in absorption cross section is $5 \times 10^{-17} \mathrm{~cm}^{2}$. At the highest stress, the energy gap is lowered around $0.1 \mathrm{eV}$. The band-edge modulation will then be significant for $\hbar \omega \geq 0.60 \mathrm{eV}$. The band-edge absorption becomes highly anisotropic with stress ${ }^{25}$ such that it is around four times larger for light polarized parallel to stress than for light polarized perpendicular to stress. Correspondingly, the thermal band-edge modulation mostly affects $\Delta \sigma_{\|}$.

\section{DISTRIBUTION FUNCTION}

To interpret the spectra, we have used an analytical form for the hot-carrier distribution function based on the drifted Maxwellian introduced by Hammar and Weissglas. ${ }^{26}$ We shall here mainly consider band 1 for which the assumed distribution function is

$$
f_{1}(\overrightarrow{\mathrm{k}})=\exp \left[-\left(\epsilon_{1}(\overrightarrow{\mathrm{k}})-\hbar \overrightarrow{\mathrm{v}}_{d} \cdot \overrightarrow{\mathrm{k}}\right) / k_{B} T\right],
$$

where $\overrightarrow{\mathrm{v}}_{d}$ is the drift velocity of carriers in band 1 and $T$ is their temperature. For convenience, hole energies are here taken to be positive with zero at the $\overrightarrow{\mathrm{k}}=0$ energy. An important property of the distribution function (5) is that the meancarrier velocity $\langle\vec{v}\rangle$ equals $\vec{v}_{d}$ for an arbitrary band structure. An optical experiment can in principle only yield information about the even part of the distribution function. However, by using the experimental drift velocity in Eq. (5), the distribution function will have the correct first moment.

Previous experiments ${ }^{4,5}$ and calculations ${ }^{17,18}$ have shown that the strong interaction between holes and optical phonons in $\mathrm{Ge}^{27}$ creates different temperatures of hot carriers with energies less than and larger than the optical-phonon energy $k_{B} \theta$ of 37 meV. We shall therefore assume different temperatures in these two regions of hole energy. Our two-temperature drifted Maxwellian is given by

$$
\begin{array}{ll}
f=\exp \left[-\left(\epsilon_{1}(\overrightarrow{\mathrm{k}})-\hbar \overrightarrow{\mathrm{v}}_{d^{\prime}} \cdot \overrightarrow{\mathrm{k}}\right) / k_{B} T_{1}\right], & \epsilon_{1}(\overrightarrow{\mathrm{k}}) \leq k_{B} \theta \\
f=A(\overrightarrow{\mathrm{k}}) \exp \left[-\left(\epsilon_{1}(\overrightarrow{\mathrm{k}})-\hbar \overrightarrow{\mathrm{v}}_{d} \cdot \overrightarrow{\mathrm{k}}\right) / k_{B} T_{2}\right], & \epsilon_{1}(\overrightarrow{\mathrm{k}})>k_{B} \theta .
\end{array}
$$

The function $A(\overrightarrow{\mathrm{k}})$ provides for the continuity of $f$ at the optical-phonon energy. The surface of con- 
stant energy equal to $k_{B} \theta$ is found from the equation $\epsilon_{1}\left(\overrightarrow{\mathrm{k}}_{0}\right)=k_{B} \theta$, where $\overrightarrow{\mathrm{k}}_{0}=\overrightarrow{\mathrm{k}}_{0}(\overrightarrow{\mathrm{k}})$ is dependent on the direction of $\vec{k}$. With this notation,

$$
A(\overrightarrow{\mathrm{k}})=\exp \left[\left(k_{B} \theta-\hbar \overrightarrow{\mathrm{v}}_{d} \cdot \overrightarrow{\mathrm{k}}_{0}\right)\left(1 / k_{B} T_{2}-1 / k_{B} T_{1}\right)\right] .
$$

For zero stress, the surface of constant heavyhole energy equal to $k_{B} \theta$ was approximated by a sphere. In the case of a uniaxial stress in the $\langle 111\rangle$ direction, we note that in a plane perpendicular to stress through $\overrightarrow{\mathrm{k}}=0$, we effectively have a sixfold symmetry which to a good approximation makes the energy isotropic in this place. It is therefore sufficient to account for the variation of $\overrightarrow{\mathrm{k}}_{0}(\overrightarrow{\mathrm{k}})$ in a plane containing the $\langle 111\rangle$ and $\langle 1 \overline{1} 0\rangle$ directions. The surface of constant energy equal to $k_{B} \theta$ was then approximated by an ellipsoid with principal axes in those directions. The axes were chosen in a way to make the ellipsoid enclose the same volume in $\vec{k}$ space as the actual surface of constant energy.

In a preliminary analysis using the parameters $T_{1}$ and $T_{2}$ to give agreement between the calculated and experimental change in absorption, we found that the anisotropy of the spectra could not be adequately reproduced unless $T_{1}$ was taken to be anisotropic. Let $\phi$ be the angle between the electric field and a point in $\overrightarrow{\mathrm{k}}$ space. The temperature $T_{1}(\phi)$ used at this point was then given by

$$
1 / T_{1}(\phi)=\cos ^{2} \phi / T_{11}+\sin ^{2} \phi / T_{1},
$$

where $T_{\text {|| }}$ is the longitudinal and $T_{\perp}$ is the transverse carrier temperature. When in Eq. (5) $T$ is $\overrightarrow{\mathrm{k}}$ dependent, the relation $\langle\overrightarrow{\mathrm{v}}\rangle=\overrightarrow{\mathrm{v}}_{d}$ is not fulfilled. For the temperatures determined here, a numerical calculation of the mean velocity gave a maximum disagreement from $\vec{v}_{d}$ of $15 \%$.

The modulation of absorption with field contains little information about carriers with large energies (Figs. 1 and 2). It was furthermore shown in Sec. V that the thermal modulation in the band edge obscures the region of high photon energies. This will mainly influence the parameter $T_{2}$. To make a better determination of $T_{2}$, we have calculated the power loss per carrier to optical phonons. In the region of fields of interest here, practically all the power input from the field, $e \overrightarrow{\mathrm{v}}_{d} \cdot \overrightarrow{\mathrm{E}}$ per carrier is given off to optical phonons. ${ }^{5}$ Let the average time between scattering processes between a carrier of energy $\epsilon(\overrightarrow{\mathrm{k}})$ and an optical phonon be $\tau_{e}(\epsilon)$ and $\tau_{a}(\epsilon)$, for emission and absorption processes respectively. The power loss to optical phonons is then given by

$$
P_{\text {opt }}=k_{B} \theta \int\left(\frac{1}{\tau_{e}}-\frac{1}{\tau_{a}}\right) f(\overrightarrow{\mathrm{k}}) d \overrightarrow{\mathrm{k}},
$$

with $f(\overrightarrow{\mathrm{k}})$ normalized. The transition probability for scattering from an initial to a final state by optical modes is first taken to be a constant. According to Conwell, ${ }^{28}$ the scattering times $\tau_{e}$ and $\tau_{a}$ are related to the density of states of the holes $D(\epsilon)$ by

$$
\frac{1}{\tau_{e}(\epsilon)} \propto(n+1) D(\epsilon-k \theta), \quad \frac{1}{\tau_{a}(\epsilon)} \propto n D(\epsilon+k \theta),
$$

where $n$ is the occupation number for optical phonons at the lattice temperature. The numerical factors entering in this expression are known from the work of Brown and Bray ${ }^{27}$ at zero stress. The transition probability for optical-mode scattering is actually dependent on the directions of initialand final-state wave vectors. ${ }^{29,30}$ When a stress is applied, wave-vector dependence of the transition probability is introduced. Using an extension to the stressed case by Lawaetz ${ }^{31}$ of Bir and Pikus' ${ }^{29}$ result for the transition probability, we found a typical average increase in this quantity of $10-15 \%$ at the highest stress. This was neglected in the calculations of the power loss. The density of states was generated in the form of histogram. The expression (8) could then be evaluated at nonzero stress. The power loss $P_{\text {opt }}$ is strongly dependent on the fraction of holes which have energies larger than $k_{B} \theta . \quad T_{2}$ was therefore adjusted to make $P_{\text {opt }} \simeq e \overrightarrow{\mathrm{v}}_{d} \cdot \overrightarrow{\mathrm{E}}$ to within $10 \%$. $P_{\text {opt }}$ is strongly dependent on $T_{2}$. The mean carrier energy is dependent on all the parameters. Consequently, this procedure will give a satisfactory determination of the mean energy.

The method used to determine the parameters of the distribution function $f\left(T_{\|}, T_{1}, T_{2}, \overrightarrow{\mathrm{v}}_{d}, \overrightarrow{\mathrm{k}}\right)$ was the following: (i) $\overrightarrow{\mathrm{v}}_{d}$ was usually the experimentally determined drift velocity. (ii) $T_{\|}$was put equal to $T_{\perp}$ and this temperature was adjusted to give an

\begin{tabular}{|c|c|c|c|c|c|}
\hline & $\begin{array}{c}X=0 \\
\left(\mathrm{~kg} / \mathrm{cm}^{2}\right)\end{array}$ & $\begin{array}{r}X=5900 \\
\left(\mathrm{~kg} / \mathrm{cm}^{2}\right)\end{array}$ & $\begin{array}{l}X=8850 \\
\left(\mathrm{~kg} / \mathrm{cm}^{2}\right)\end{array}$ & $\begin{array}{l}X=11800 \\
\left(\mathrm{~kg} / \mathrm{cm}^{2}\right)\end{array}$ & $\begin{array}{c}E \\
(\mathrm{~V} / \mathrm{cm})\end{array}$ \\
\hline$v_{d}$ expt. & & & & 0.60 & \\
\hline$v_{d}$ calc. & & & & $\cdots$ & \\
\hline$T_{4}$ & & & & 130 & 100 \\
\hline$T_{\perp}$ & & & & 130 & \\
\hline$T_{2}$ & & & & 65 & \\
\hline$v_{d}$ expt. & 0.42 & 0.75 & 1.00 & 1.20 & \\
\hline$v_{d}$ calc. & $0.32^{a}$ & $\cdots$ & $\cdots$ & $\cdots$ & \\
\hline$T_{11}$ & 140 & 150 & 130 & 120 & 250 \\
\hline$T_{\perp}$ & 135 & 150 & 150 & 160 & \\
\hline$T_{2}$ & 50 & 60 & 50 & 60 & \\
\hline$v_{d}$ expt. & 0.66 & 1.05 & 1.30 & 1.62 & \\
\hline$v_{d}$ calc. & $\cdots$ & $\cdots$ & 1.00 & 1.20 & \\
\hline$T_{\|}$ & 100 & 250 & 200 & 200 & 500 \\
\hline$T_{\perp}$ & 160 & 250 & 250 & 200 & \\
\hline$T_{2}$ & 70 & 70 & 70 & 85 & \\
\hline$v_{d}$ expt. & 0.90 & 1.32 & 1.47 & & \\
\hline$v_{d}$ calc. & $\cdots$ & $\cdots$ & 1.20 & & \\
\hline$T_{11}$ & 120 & 300 & 500 & & 1000 \\
\hline$T_{\perp}$ & 180 & 300 & 500 & & \\
\hline$T_{2}$ & 85 & 85 & 110 & & \\
\hline
\end{tabular}

TABLE II. Distribution function parameters. $v_{d}$ is in units of $10^{7} \mathrm{~cm} / \mathrm{sec}$ and $T_{\|}, T_{\perp}$, and $T_{2}$ are in units of ${ }^{\circ} \mathrm{K}$.

${ }^{a}$ Estimated contribution from heavy holes. 


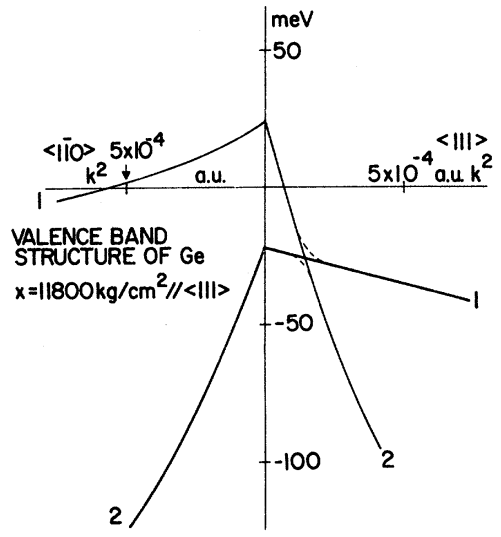

FIG. 11. Four-band calculation of the band structure of the $p_{3 / 2}$ levels in the $\langle 111\rangle$ and $\langle 1 \overline{1} 0\rangle$ directions for a stress of $11800 \mathrm{~kg} / \mathrm{cm}^{2}$ in the $\langle 111\rangle$ direction. The dashed line illustrates the behavior in a direction deviating slightly from the $\langle 111\rangle$ direction. The uninteresting degeneracy at the two points in the $\langle 111\rangle$ direction has now been removed. The numbers 1 and 2 denote the levels as defined in Sec. 2.

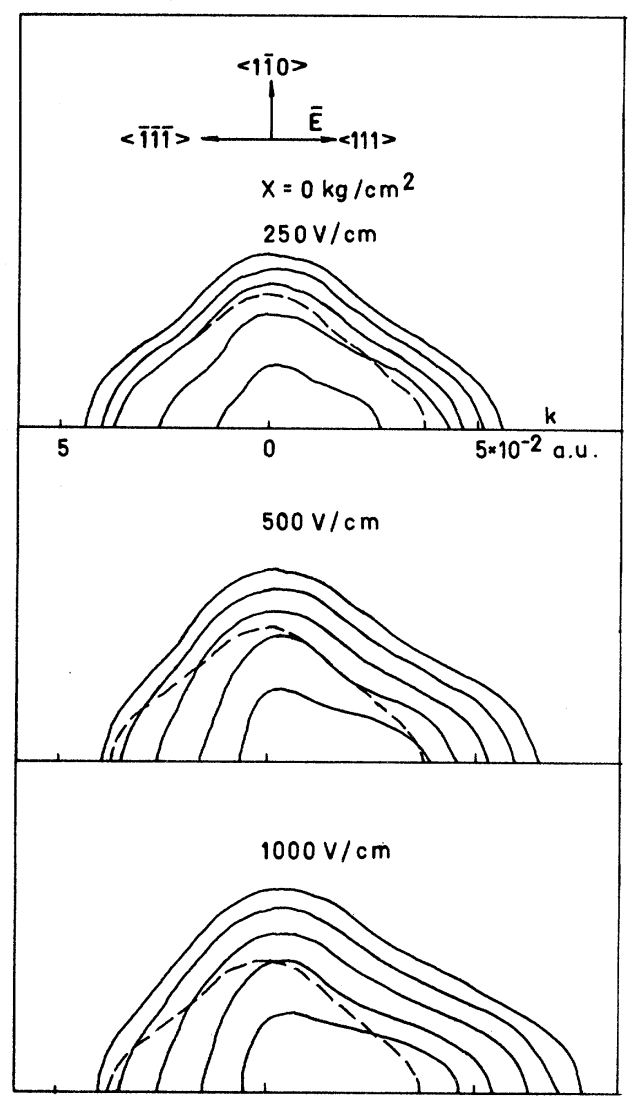

FIG. 12. Curves of constant occupation probability for different fields at zero stress. The occupation probability is normalized to 1 in the center and the curves represent probabilities of $0.5,10^{-1}, 10^{-2}, 10^{-3}$, and $10^{-4}$. The dashed line gives the position of the optical-phonon energy. approximate agreement with the experimental change in absorption at the maximum $\Delta \sigma$ around $\hbar \omega=0.42 \mathrm{eV}$. (iii) $T_{\|}$and $T_{1}$ were adjusted to give the experimental anisotropy, i.e., the correct values of $\Delta \sigma_{\|}$and $\Delta \sigma_{\perp}$ at the maximum. (iv) $T_{2}$ was adjusted to give agreement between the calculated power loss to optical modes and the input power from the field.

The results of this procedure are shown in Table II. At zero stress and $1000 \mathrm{~V} / \mathrm{cm}, T_{2}$ is equal to the lattice temperature, in agreement with the results of Ref. 4.

The change in absorption calculated with these parameters is shown in Figs. 6-9 together with the experimental results. The general agreement between the experimental and calculated spectra is rather good. In the region of high photon energies, the agreement is usually better for $\Delta \sigma_{\perp}$ than for $\Delta \sigma_{11}$ as anticipated in Sec. V. The calculated spectra account only for the contribution from 1-3 transitions. Although the parameters $T_{11}$ and $T_{1}$ were determined to give agreement at the maximum $\Delta \sigma$, there is in most cases a rather good agreement also at the minimum around $\hbar \omega=0.33 \mathrm{eV}$.

At the highest fields and stresses it was not pos-

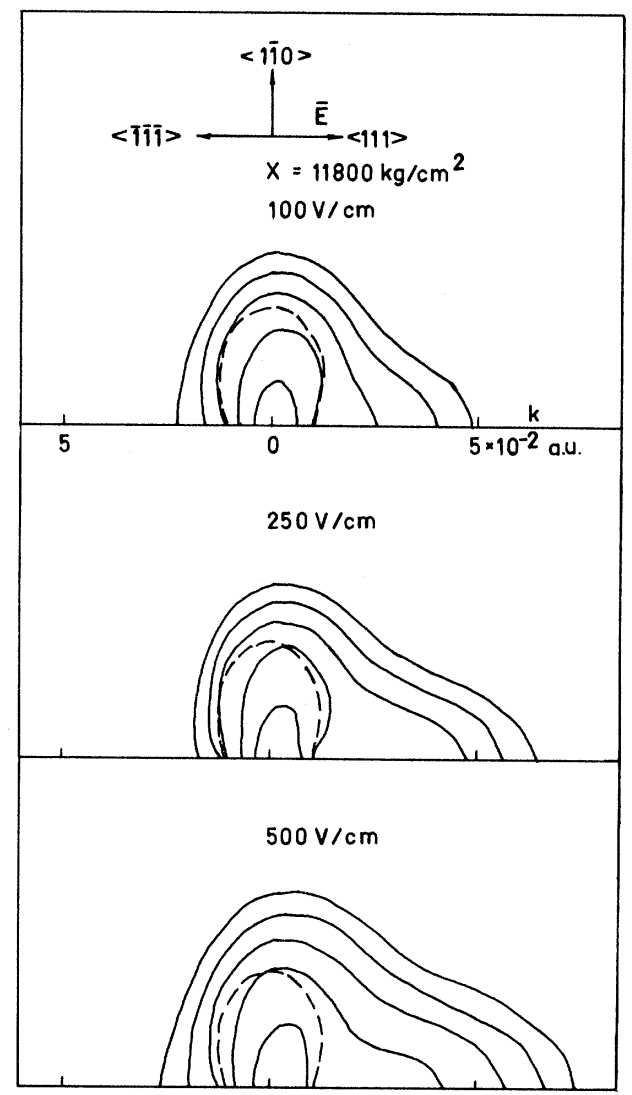

FIG. 13. Same as Fig. 12 but for a stress of 11800 $\mathrm{kg} / \mathrm{cm}^{2}$ in the $\langle 111\rangle$ direction. 


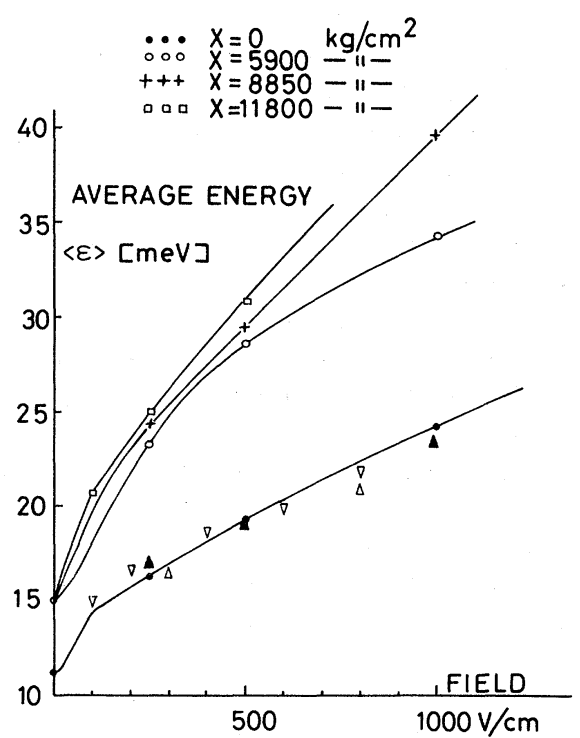

FIG. 14. Mean energies of carriers vs field for different uniaxial stresses in the $\langle 111\rangle$ direction. Lattice at $85^{\circ} \mathrm{K} . \Delta$ : Pinson and $\mathrm{Bray}^{5} ; \nabla$ : Kurosawa ${ }^{17}$; $\Delta$ : Budd. ${ }^{18}$

sible to obtain agreement using the experimentally determined drift velocity. This inevitably gave a $\Delta \sigma_{\perp}$ which was too large relative to $\Delta \sigma_{\|}$. In this case, we let $T_{\|}=T_{\perp}$ and adjusted $\overrightarrow{\mathrm{v}}_{d}$ to obtain the proper anisotropy in the spectra. A distribution function determined in this way will still describe the even properties of the actual distribution function. The streaming character of the carrier motion is illustrated by the surfaces of constant occupation probability shown in Figs. 12 and 13. The extent of the curves in $\overrightarrow{\mathrm{k}}$ space is comparable for zero stress, $1000 \mathrm{~V} / \mathrm{cm}$ and for $11800 \mathrm{~kg} / \mathrm{cm}^{2}$, $500 \mathrm{~V} / \mathrm{cm}$ although in the latter case the carriers have to overcome a "potential barrier" of $45 \mathrm{meV}$ (see Fig. 11) to reach that far out.

The lower of the strain-split $p_{3 / 2}$ bands gives a contribution to the absorption for photon energies smaller than $0.29 \mathrm{eV}$. To get some feeling with the properties of holes in this band, we have at $8850 \mathrm{~kg} / \mathrm{cm}^{2}$ and $1000 \mathrm{~V} / \mathrm{cm}$, used the distribution function for this band

$$
f_{2}=Z \exp \left[-\left(\epsilon_{2}(\overrightarrow{\mathrm{k}})-\hbar \overrightarrow{\mathrm{v}}_{d} \cdot \overrightarrow{\mathrm{k}}\right) / k_{B} T\right]
$$

where $Z$ determines the fraction of carriers in band 2. To get a maximum in differential absorption at $0.27 \mathrm{eV}$, a carrier temperature $\geq 200^{\circ} \mathrm{K}$ was needed. One percent of the carriers placed in band 2 produced an absorption cross section of $2 \times 10^{-17}$ to $4 \times 10^{-17} \mathrm{~cm}^{2}$ at $0.27 \mathrm{eV}$. The calculated ratio $\Delta \sigma_{\|}: \Delta \sigma_{\perp}=1: 1.4$ was only weakly dependent on the drift velocity used in Eq. (10).

\section{SOME HIGH-FIELD PROPERTIES OF UNIAXIALLY STRESSED $p$-TYPE Ge}

\section{A. Application of Distribution Functions}

In this section we discuss some hot-carrier properties of uniaxially stressed $p$-type Ge. The basis for the discussion is the average kinetic energy of carriers in band 1 show $n$ in Fig. 14. The average energy was calculated by numerical integration using the parameters for the distribution functions given in Table II. For zero stress we can compare those results with other published material. ${ }^{5,17,18}$ In general, there is good agreement. Our value at $1000 \mathrm{~V} / \mathrm{cm}$ is perhaps too large corresponding to the overestimate of $\Delta \sigma_{\perp}$ in Fig. 6 . When a stress is applied, the mean energy is increased. At low fields this increase is roughly independent of stress. At high fields, the energies for zero stress and for $5900 \mathrm{~kg} / \mathrm{cm}^{2}$ have the same asymptotic field dependence, which is different from the asymptotic behavior at the two highest stresses. The mean energy can be related to the power input per carrier from the field by

$$
e \overrightarrow{\mathrm{v}}_{d} \cdot \overrightarrow{\mathrm{E}}=\left(\langle\epsilon\rangle-\langle\epsilon\rangle_{T}\right) / \tau_{\epsilon},
$$

where $\langle\epsilon\rangle_{T}$ is the mean energy in thermal equilibrium at $85^{\circ} \mathrm{K} . \tau_{\epsilon}$ shown in Fig. 15 , is the mean energy gained per unit power input. It is not in general possible to associate $\tau_{\epsilon}$ with an energy relaxation time. At high fields, $\tau_{\epsilon}$ approaches the same value for zero stress and for $5900 \mathrm{~kg} / \mathrm{cm}^{2}$. The conversion of power to mean energy is less efficient in stressed material than in unstressed except at rather high fields and stresses.

In order to give an interpretation of those results, we consider the mean time between scatterings at hole energy $\epsilon$. For optical-mode scatter-

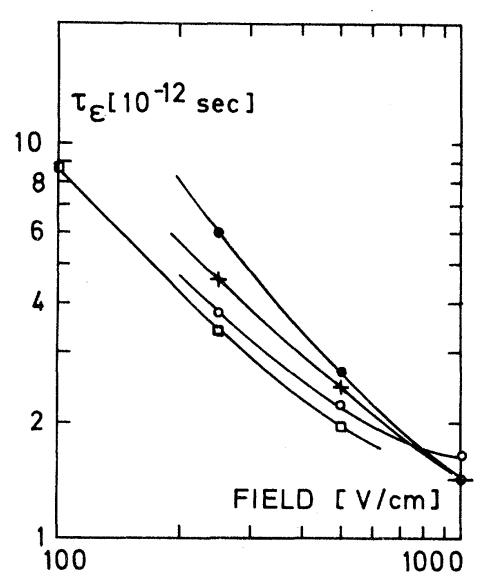

FIG. 15. "Energy relaxation time" vs field at $85^{\circ} \mathrm{K}$. $\bullet,+, O$, and $\square$ denote stresses of $0,5900,8850$, and $11800 \mathrm{~kg} / \mathrm{cm}^{2}$ in the $\langle 111\rangle$ direction. 
ing, this time is only dependent on the density of final states available for the scattered carrier [Eq. (9)]. For a nondegenerate band in an isotropic medium, the mean time between collisions with acoustical phonons is ${ }^{28}$

$$
\frac{1}{\tau_{\mathrm{ac}}(\epsilon)} \propto D(\epsilon) \text {. }
$$

This expression is based on equipartition and negligible energy of the acoustical phonons. $\tau_{\text {ac }}$ is the resulting value for emission and absorption processes. For the complex valence-band structure of uniaxially stressed Ge, we shall assume that Eq. (12) is still qualitatively correct.

The density of states is shown in Fig. 16 for zero stress and for $11800 \mathrm{~kg} / \mathrm{cm}^{2}$. For energies below the strain splitting, the density of states is diminished 2-8 times by stress. The mean collision times for the different scattering processes have been given in Refs. 5 and 27 for unstressed material. For a given stress, the collision times at a given carrier energy can be found from the values at zero stress using the relations (9) and (12). Figure 17 shows the mean collision time for acoustical-mode scattering $\tau_{\mathrm{ac}}(\epsilon)$ and for opticalmode scattering $\tau_{\text {opt }}(\epsilon)$. Impurity scattering was not taken into account. The density of states at some energy $\epsilon$ governs the acoustical-mode scattering at this carrier energy, the optical-phonon absorption at energy $\epsilon-k_{B} \theta$, and optical-phonon emission at energy $\epsilon+k_{B} \theta$. Calling the strain splitting of the valence bands $\delta$, the stress behavior of the scattering processes can be summarized as follows: (i) Scattering rate by acoustical modes is

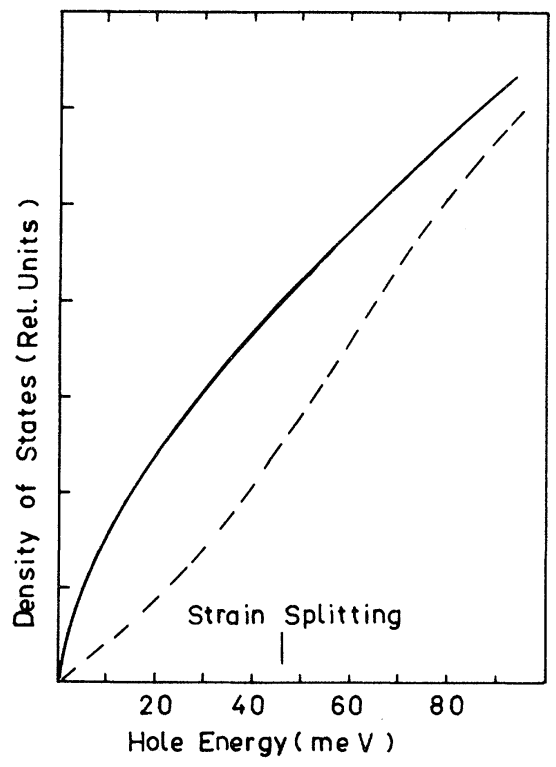

FIG. 16. Density of states. Full line is at zero stress and dashed line at $11800 \mathrm{~kg} / \mathrm{cm}^{2}$ in the $\langle 111\rangle$ direction.
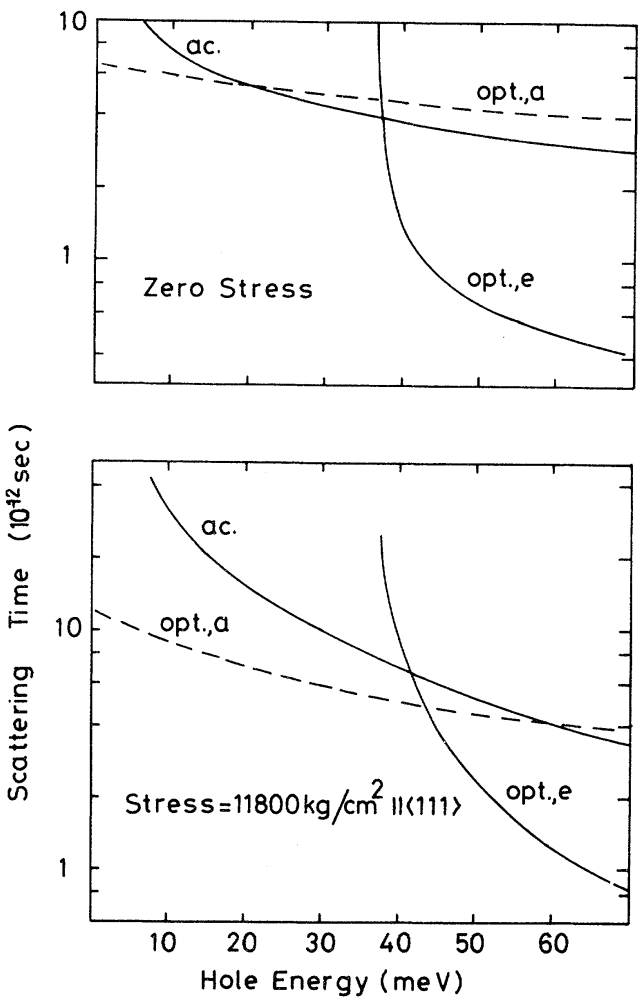

FIG. 17. Mean time between scatterings vs hole energy at $85^{\circ} \mathrm{K}$. Upper set of curves is at zero stress, lower at a stress of $11800 \mathrm{~kg} / \mathrm{cm}^{2}$ in the $\langle 111\rangle$ direction.

decreased for $\epsilon<\delta$ and is approximately unchanged for $\epsilon>\delta$. (ii) Scattering rate by absorption of optical phonons is almost independent of stress.

(iii) Scattering rate by emission of optical phonons is strongly diminished in the energy range $k_{B} \theta<\epsilon$ $<k_{B} \theta+\delta$.

The carriers have a cycle of streaming motion ${ }^{5,32}$ consisting of: (a) an initial acceleration through the $\overrightarrow{\mathrm{k}}$ space region where $\epsilon<k_{B} \theta$. (b) The carrier obtains an energy larger than the optical-phonon energy and emits an optical phonon whereupon the cycle is repeated. The time needed for a carrier to be accelerated from $\overrightarrow{\mathrm{k}}=0$ to the optical-phonon energy is dependent on stress. Considering the band structure in the $\langle 111\rangle$ direction (Fig. 11), we see that the band structure at $\epsilon=k_{B} \theta$ is heavy holelike for $\delta<k_{B} \theta$ and light holelike for $\delta \geq k_{B} \theta$. In a field of $1000 \mathrm{~V} / \mathrm{cm}$, the time it takes for a carrier to be accelerated from $\overrightarrow{\mathrm{k}}=0$ to the opticalphonon energy is $\simeq 5$ psec at zero stress and 1-2 psec at high stresses. This, together with (i) makes the first part of the cycle of streaming motion go faster when a stress is applied. On the other hand, the second part of the cycle will slow down due to (iii). As a result of the stress, the carriers will spend less time in the region $\epsilon<k_{B} \theta$ 
and more time in the region $\epsilon>k_{B} \theta$. The average energy, therefore, increases with stress. For a given strain splitting $\delta$, the effect of further stress is only to change the density of states at energies above $\delta$. Thus, when $\delta>k_{B} \theta$, an increase of stress changes the emission rate of optical phonons only at carrier energies larger than $k_{B} \theta+\delta$. For strain splittings above $k_{B} \theta$, the only change in collision times occur at energies larger than $k_{B} \theta+\delta$. The mean energy will therefore approximately saturate for strain splitting above $k_{B} \theta$, corresponding to a stress of $8850 \mathrm{~kg} / \mathrm{cm}^{2}$. At large hole energies, the scattering probabilities approach the values at zero stress. As a consequence, the mean energy of the carriers obtains the same asymptotical behavior as for zero stress. In the range of fields used, this limit is only obtained for the smallest stress.

\section{B. Discussion of BNDC in Uniaxially Stressed $p$-type Ge}

In this section, we discuss some possible mechanisms for the occurrence of BNDC in uniaxially compressed $p$-type Ge. BNDC occurs only above a certain stress level, which is dependent on doping ${ }^{8}$ and temperature. ${ }^{9}$ At $4.2^{\circ} \mathrm{K}$, the threshold field increases with stress ${ }^{8}$ whereas at $27^{\circ} \mathrm{K}$ and above, the threshold field decreases with stress. ${ }^{9}$ We shall only consider the latter case.

The band structure of Fig. 11 is for small wave vectors composed of two strain-split valleys centered at $\overrightarrow{\mathrm{k}}=0$. The masses of the upper and lower valley in the field direction are given by $\left(m_{0} / m_{1}\right)_{111}$ $=\left|A+\frac{1}{3} N\right| \simeq 25$ and $\left(m_{0} / m_{2}\right)_{111}=\left|A-\frac{1}{3} N\right| \simeq 2$. These masses are valid over energy ranges of $\delta$ and $\frac{2}{25} \times \delta$, respectively. For energies outside this range, the masses are interchanged.

Ridley and Watkins ${ }^{11}$ initially considered the mechanism of carrier transfer between the valleys around $\overrightarrow{\mathrm{k}}=0$. In order to get a BNDC, the carriers in valley 2 must experience a heavy mass. At strain splittings necessary to obtain BNDC around $40 \mathrm{meV},{ }^{9}$ the mass in valley 2 is light holelike for energies larger than $3 \mathrm{meV}$ above the extremum of this band. In a field of $1000 \mathrm{~V} / \mathrm{cm}$, a carrier in band 2 is accelerated from $\overrightarrow{\mathrm{k}}=0$ to the small mass region in 1-2 psec. Typical scattering times for such a carrier are 2-5 psec (Fig. 17). The mean energy of carriers in band 2 is thus considerably larger than $3 \mathrm{meV}$ and most carriers in this band therefore experience a small mass. A BNDC at high fields is therefore not expected from the mechanism of carrier transfer between the $\vec{k}=0$ valleys.

We next consider the possibility of a BNDC based on the nonparabolicity ${ }^{10,13,26}$ of the upper valence band. The basic idea is, that the electric field transfers carriers out to a part of the band structure with large mass where they give a small con- tribution to the current. The ratio between the strain splitting necessary to produce BNDC and the optical-phonon energy $\delta / k_{B} \theta$ equals $0.95,1.1$, 1.5 , and 1.6 at $27,77,140$, and $160^{\circ} \mathrm{K}$, respectively. ${ }^{9}$ The two last values are not well defined. At $85^{\circ} \mathrm{K}$, it is therefore natural to seek an explanation of the BNDC from the case $\delta=k_{B} \theta$. Let us separate the carriers in a fraction $n_{a}$ which have energies less than the optical-phonon energy, and a fraction $n_{b}$ with energies larger than this energy. With the distribution function $f(\overrightarrow{\mathrm{k}})$ normalized to unity,

$$
\int f(\overrightarrow{\mathrm{k}}) d \overrightarrow{\mathrm{k}}=\int_{\epsilon=0}^{k_{B} \theta} f(\overrightarrow{\mathrm{k}}) d \overrightarrow{\mathrm{k}}+\int_{\epsilon=k_{B} \theta}^{\infty} f(\overrightarrow{\mathrm{k}}) d \overrightarrow{\mathrm{k}}=n_{a}+n_{b}
$$

and similar for the drift velocity

$$
\begin{aligned}
\overrightarrow{\mathrm{v}}_{d} & =\langle\overrightarrow{\mathrm{v}}\rangle=\int \overrightarrow{\mathrm{v}}(\overrightarrow{\mathrm{k}}) f(\overrightarrow{\mathrm{k}}) d \overrightarrow{\mathrm{k}}=\int_{\epsilon=0}^{k_{B} \theta} \overrightarrow{\mathrm{v}} f d \overrightarrow{\mathrm{k}}+\int_{\epsilon=k_{B} \theta}^{\infty} \overrightarrow{\mathrm{v}} f d \overrightarrow{\mathrm{k}} \\
& =\overrightarrow{\mathrm{v}}_{a} n_{a}+\overrightarrow{\mathrm{v}}_{b} n_{b} .
\end{aligned}
$$

Carriers in part $a$ of the band are only weakly scattered by acoustical phonons and by absorption of optical phonons. This yields a high carrier temperature (Table II). The small mass in this region gives large $\vec{v}(\vec{k})$ in the first part of the integral in Eq. (14). Carriers in part $b$ of the band are strongly scattered by emission of optical phonons. This gives a small carrier temperature (Table II). The $\overrightarrow{\mathrm{v}}(\overrightarrow{\mathrm{k}})$ are small in this region owing to the large mass in the field direction. It should furthermore be mentioned that the ratio of scattering rates $1 / \tau_{\mathrm{ac}}: 1 / \tau_{\mathrm{op}, \mathrm{e}}$ increases a factor $2-3$ from zero stress to the stress where $\delta=k_{B} \theta$. Correspondingly, one would at high stresses expect a larger degree of velocity randomizing for carriers in part $b$ of the band.

In Eq. (14) we shall therefore neglect the contribution from part $b$ of the band to $\overrightarrow{\mathrm{v}}_{d}$ and let

$$
\overrightarrow{\mathrm{v}}_{d} \simeq n_{a} \overrightarrow{\mathrm{v}}_{a}
$$

A BNDC will thus occur if the rate of carrier transfer with field from part $a$ to $b$ of the band is sufficiently large. Figure 18 shows the fraction of carriers $n_{b}$ which have energies larger than the optical-phonon energy. This was calculated from the distribution functions of Table II. Basically, the field and stress dependence of $n_{b}$ is the same as of the mean energy. Using $n_{b}$ and the measured drift velocities (Table II), we have calculated $\overrightarrow{\mathrm{v}}_{a}$ from Eq. (15). The result is displayed in the lower set of curves in Fig. 19. To make a comparison between different values of stress, the same procedure was used for all stresses. The power dependence of $\vec{v}_{a}$ on field is rather constant over the range of investigation. We therefore find $\vec{v}_{a}$ at higher fields by extrapolation. The values of $n_{b}$ 


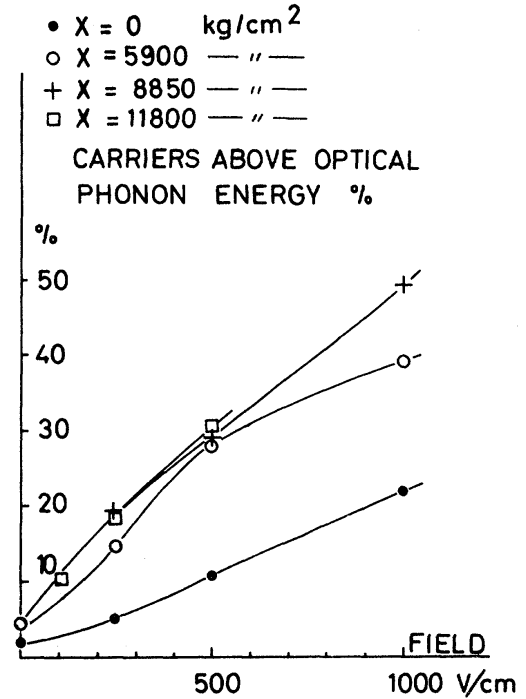

FIG. 18. Fraction of carriers which have energies larger than the optical-phonon energy shown vs field for stresses in the $\langle 111\rangle$ direction.

were extrapolated from the measured values in a narrow range of fields. The drift velocity at higher fields is found from these values as $\vec{v}_{d}$ $=\overrightarrow{\mathrm{v}}_{a}\left(1-n_{b}\right)$. The result is shown on the upper set of curves in Fig. 19. A BNDC occurs only for stresses of the investigation above $8850 \mathrm{~kg} / \mathrm{cm}^{2}$ where $\delta=k_{B} \theta$. The predicted threshold field is $\simeq 1100 \mathrm{~V} / \mathrm{cm}$ at $8850 \mathrm{~kg} / \mathrm{cm}^{2}$ and $\simeq 900 \mathrm{~V} / \mathrm{cm}$ at $11800 \mathrm{~kg} / \mathrm{cm}^{2}$. The experimental threshold field for oscillations is around $500 \mathrm{~V} / \mathrm{cm}^{9,10}$ at these values of stress. The threshold field for spikewave oscillations ${ }^{10}$ is however around $1000 \mathrm{~V} / \mathrm{cm}$. If we let $\overrightarrow{\mathrm{v}}_{b}=\frac{1}{10} \overrightarrow{\mathrm{v}}_{a}$, the BNDC persists at $8850 \mathrm{~kg} /$ $\mathrm{cm}^{2}$ and disappears at $11800 \mathrm{~kg} / \mathrm{cm}^{2}$. The rate of change of $n_{b}$ necessary to produce BNDC at 8850 $\mathrm{kg} / \mathrm{cm}^{2}$ and $1100 \mathrm{~V} / \mathrm{cm}$ is $\simeq 3 \% / 100 \mathrm{~V} / \mathrm{cm}$. This justifies the use of extrapolation of $n_{b}$ to higher fields.

\section{SUMMARY}

The modulation of intervalence-band absorption of infrared light by pulsed electric field has been studied for fields $\leq 1000 \mathrm{~V} / \mathrm{cm}$ and uniaxial stress $\leq 11800 \mathrm{~kg} / \mathrm{cm}^{2}$. All measurements were performed with electric field and uniaxial stress along the $\langle 111\rangle$ direction. The modulation is strongly dependent on the polarization of light with respect to the field direction and of the magnitude of stress.

The main objective was to determine the hotcarrier distribution function in $\overrightarrow{\mathrm{k}}$ space. The analysis of the results was based on (a) a numerical calculation of the intervalence-band absorption and (b) a parametrized distribution function for the hot carriers. The calculational procedure (a) was checked by measurements of piezoabsorption in thermal equilibrium. The parameters of the distribution function (b) were varied until the calculated modulation of absorption agreed with the measured one. In order to reproduce the distribution function at high energies, one of the parameters was adjusted to give agreement between the measured power loss $e \overrightarrow{\mathrm{v}}_{d} \cdot \overrightarrow{\mathrm{E}}$ and the calculated rate of energy loss to optical phonons. Only the effect of the upper of the strain-split valence bands was taken into account in the calculation. The main results can be summarized as follows.

(i) The hot-carrier distribution function is strongly anisotropic in $\vec{k}$ space. The nonparabolicity of the band structure when a stress is applied further increases this anisotropy.

(ii) The effect of stress is to relax the acoustical-scattering probability for $\epsilon<\delta$, and the probability for emission of optical phonons in the region $k_{B} \theta \leq \epsilon \leq k_{B} \theta+\delta$. This results in an increase of the mean-carrier energy and in an increased transfer of carriers to the region $\epsilon \geq k_{B} \theta$.

(iii) The relaxation in scattering probabilities saturates when the strain splitting reaches the optical-phonon energy. Further stress mainly affects carriers with $\epsilon>\delta+k_{B} \theta$.

The two latter features can essentially be understood from the stress dependence of the density of states. This is strongly diminished for $\epsilon<\delta$.

A simple model for the BNDC occurring in uni-

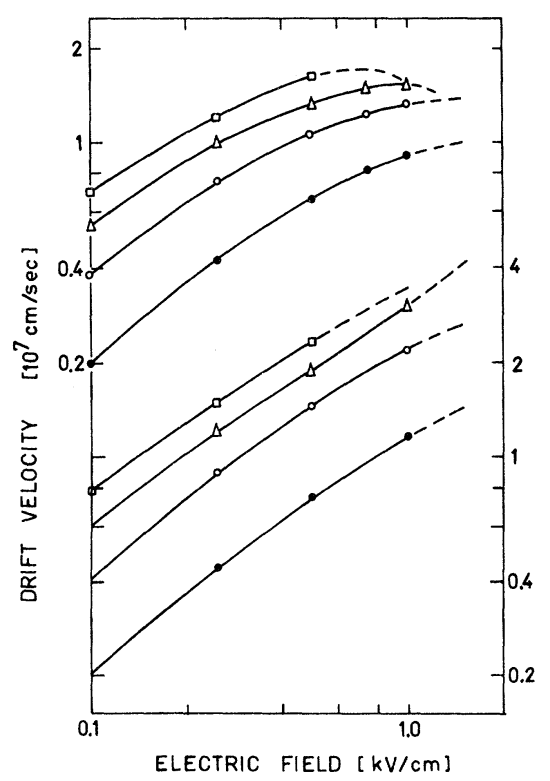

FIG. 19. Drift velocities of all carriers (upper set of curves, left scale), and of carriers with $\epsilon \leq k_{B} \theta$ (lower set of curves, right scale). $\bullet, 0, \Delta$, and $\square$ denote stresses of $0,5900,8850$, and $11800 \mathrm{~kg} / \mathrm{cm}^{2}$ in the $\langle 111\rangle$ direction. 
axially stressed $p$-type Ge has been proposed. When a stress is applied, a significant fraction of carriers is transferred to the region $\epsilon>\delta$ with large mass. When $\delta=k_{B} \theta$, the carriers in this region emit optical phonons which decreases the carrier temperature in the region $\epsilon>k_{B} \theta$. This region thus gives a very small contribution to the drift velocity. The BNDC then arises if the field rate of carrier transfer to $\epsilon>k_{B} \theta$ is sufficiently large.

These results suggest that a theoretical treatment of high-field effects in highly stressed $p$-type
Ge can be simplified by using a two-level system, where the level separation is at the optical-phonon energy.

\section{ACKNOWLEDGMENTS}

The author is grateful to Professor N. I. Meyer for his continuous interest in this work and to the Northern Europe University Computing Center (NEUCC) for supplying a considerable amount of computer time. During the course of this work, the author has benefited greatly from discussions with Professor I. Balslev.

\footnotetext{
* Present address: IBM Watson Research Center, Yorktown Heights, N. Y. 10598.
}

${ }^{1}$ M. A. S. C. Brown and E. G. S. Paige, Phys. Rev. Letters $\underline{7}, 84$ (1961).

${ }^{2}$ M. A. S. C. Brown, E. G. S. Paige, and L. N. Simcox, in Proceedings of the International Conference on Semiconductor Physics, Exeter, England, edited by A. C. Stickland (The Institute of Physics and the Physical Society, London, 1962), p. 111.

${ }^{3}$ R. Bray and W. Pinson, Phys. Rev. Letters 11, 268 (1963).

${ }^{4}$ A. C. Baynham and E. G. S. Paige, Phys. Letters $\underline{6}$, 7 (1963)。

${ }^{5}$ W. E. Pinson and R. Bray, Phys. Rev. 136, A1449 (1964).

${ }^{6}$ A. C. Baynham and E. G. S. Paige, in Proceedings of the 7th International Conference on the Physics of Semiconductors, Paris, France, 1964 (Academic, New York, 1964), p. 150.

${ }^{7}$ W. Faw cett, Proc. Phys. Soc. (London) 85, 931 (1965).

${ }^{8}$ A. A. Kastal'skii and S. M. Ryvkin, Fiz. Tekh. Poluprov. 1, 622 (1967) [Sov. Phys. Semicond. 1, 523 (1967)].

${ }^{9}$ J. E. Smith, Jr., J. C. McGroddy, and M. I. Nathan, Phys. Rev. 186, 727 (1969).

${ }^{10}$ N. O. Gram and N. I. Meyer, Phys. Status Solidi $\underline{1}$, 237 (1971).

${ }^{11}$ B. K. Ridley and T. B. Watkins, Proc. Phys. Soc. (London) 78, 293 (1961).

${ }^{12}$ G. E. Pikus and G. L. Bir, Fiz. Tverd. Tela 1 , 1942 (1959) [Sov. Phys. Solid State 1, 1502 (1960)].

${ }^{13}$ D. Matz, J. Phys. Chem. Solids 28,373 (1967).
${ }^{14}$ J. B. Gunn, Solid State Commun. 1,89 (1963).

${ }^{15}$ G. S. Hobson and E. G. S. Paige, in Ref. 6, p. 144.

${ }^{16}$ I. Balslev, Phys. Rev. 177, 1173 (1969).

${ }^{17}$ T. Kurosawa, J. Phys. Soc. Japan Suppl. 21, 424 (1966)。

${ }^{18}$ H. Budd, Phys. Rev. 158, 798 (1967).

${ }^{19}$ A. H. Kahn, Phys. Rev. 97, 1647 (1955).

${ }^{20}$ E. O. Kane, J. Phys. Chem. Solids 1,82 (1956).

${ }^{21} \mathrm{~J}$. B. A rthur, A. C. Baynham, W. Fawcett, and E.

G. S. Paige, Phys. Rev. 152, 740 (1966).

${ }^{22} \mathrm{~T}$. P. McLean, in Progress in Semiconductors, edited by A. F. Gibson (Heywood, London, 1960), Vol. 5.

${ }^{23}$ G. Dresselhaus, A. F. Kip, and C. Kittel, Phys. Rev. 98, 368 (1955).

${ }^{24}$ G. G. Macfarlane, T. P. McLean, J. E. Quarrington, and V. Roberts, Phys. Rev. 108, 1377 (1957).

${ }^{25} \mathrm{E}$. Alder and E. Erlback, Phys. Rev. Letters 16 , 87 (1966); S. Riskaer and I. Balslev, Phys. Letters 21, 16 (1966).

${ }^{26}$ C. Hammar and P. Weissglas, Phys. Status Solidi 24 , 531 (1967).

${ }^{27}$ D. M. Brown and R. Bray, Phys. Rev. 127, 1593 (1962).

${ }^{28}$ E. M. Conwell, in Solid State Physics, edited by F. Seitz and D. Turnbull (Academic, London, 1967), Suppl. 9.

${ }^{29}$ G. L. Bir and G. E. Pikus, Fiz. Tverd. Tela 2 , 2287 (1960) [Sov. Phys. Solid State 2, 2039 (1961)].

${ }^{30}$ P. Lawaetz, Phys. Rev。 174,867 (1968).

${ }^{31} \mathrm{P}$. Lawaetz (private communication).

${ }^{32}$ W. Shockley, Bell System Tech. J. 20, 990 (1951)。 